\title{
Probing Macromolecular Dynamics and the Influence of Finite Size Effects
}

\author{
SCOTT Sills AND RENÉ M. OVERNEY \\ Department of Chemical Engineering, University of Washington, Box 351750, Seattle, WA 98195-1750, USA
}

\begin{tabular}{|c|c|c|}
\hline \multicolumn{3}{|c|}{ Contents } \\
\hline & Introduction & 2 \\
\hline 2. & The Glass Transition and Molecular Mobility & 2 \\
\hline & Macromolecular Probing Techniques & 5 \\
\hline & 3.1 Static Contacts & 5 \\
\hline & 3.2 Modulated Contacts & 6 \\
\hline & 3.3 Calibration of Lateral Forces in Scanning Probe Microscopy & 7 \\
\hline & 3.4 Shear Modulation Force Microscopy & 8 \\
\hline & 3.5 Friction Force Microscopy & 9 \\
\hline & 3.6 Tribological Models for Friction Force Microscopy & 10 \\
\hline & Internal Friction and Dynamics near the Glass Transition & 12 \\
\hline & 4.1 Molecular Relaxations & 12 \\
\hline & 4.2 Structural Heterogeneity & 14 \\
\hline & 4.3 Cooperative Molecular Motion & 15 \\
\hline & Constraints and Structural Modifications near Interfaces & 16 \\
\hline & 5.1 Interfacial Plasticization & 16 \\
\hline & 5.2 Dewetting Kinetics & 16 \\
\hline & 5.3 Disentanglement Barriers & 17 \\
\hline & 5.4 Interfacial Glass Transition Profiles & 18 \\
\hline & Mechanical Operations in Nanoscopic Polymer Systems & 19 \\
\hline & 6.1 Indentation Contact Mechanics & 19 \\
\hline & 6.2 Rim Formation during Indentation & 21 \\
\hline & 6.3 Strain Shielding and Confined Plasticity & 23 \\
\hline & 6.3.1 Substrate Constraints & 23 \\
\hline & 6.3.2 Structural Anisotropy & 24 \\
\hline & Closing Remarks & 25 \\
\hline
\end{tabular}




\section{Introduction}

Miniaturization trends in electronic, mechanical, optical, and biomedical devices have brought the nanoscale to the forefront of the engineering design arena. In systems reduced to nanoscopic dimensions, bulk statistical averaging and continuum models are jeopardized. Interfacial constraints lead to bulk-deviating molecular dynamics; material and transport properties are altered. Engineering efforts must work within these constraints, or yet, utilize the constraints as design opportunities. Today's challenge lies in obtaining convenient access to the molecular mobility, using novel approaches, such as real-space scanning probe techniques. With this, we can begin to optimize molecular designs based on the critical lengths that are set by nanotechnological device applications.

Polymers and functionalized macromolecules belong to a class of materials that are particularly well suited for bottom-up, molecular design approaches. In solid state form, one of the foremost important phenomena is thus the glass transition, as it dictates the material's properties, particularly its transport properties. The glass transition is rooted in the underlying molecular mobility, which itself, is strongly influenced by nanoscopic constraints [1]. In this chapter, we focus on recent scanning probe microscopy (SPM) developments that address issues related to the glass transition in confined polymer systems. Particular emphasize is given to the local probing of molecular dynamics near the glass transition, the influence of finite size constraints on these dynamics, and the impact of the molecular mobility on current nanotechnological developments in polymer thin films.

We discuss the frictional dissipation in SPM with respect to the availability of particular molecular relaxations. In polymer melts near the glass transition, nanoscopic friction reveals a highly cooperative dissipation phenomena. This phenomena is known in the open literature as the heterogeneous dynamics of glass formers. It introduces a critical length scale, over which collective molecular motion occurs, i.e. the size of a cooperatively rearranging region (CRR). In the bulk, this dimension ranges from a collection of monomeric segments on the subnanometer scale to tens of nanometers, involving several molecules [2]. The large size of the CRRs will lead our discussion towards the impact of dimensional constraints in thin films systems. With shear modulated force microscopy (SM-FM) and friction force microscopy (FFM) techniques, we address the molecular restructuring and material anisotropy due to interfacial constraints; which can lead to plasticization near interfaces in polydisperse and heterogeneous systems, and modified dewetting kinetics and instabilities in thin film systems.

Having addressed the impact of nanoscopic constraints on local molecular mobility, we return to current technological challenges in nano-electromechanical systems (NEMS). We explore how the contact mechanics in the thin films used for terabit data storage [3] are compounded by: (i) high strain rate, nano-impact conditions, (ii) the proximity of the underlying substrate, and (iii) material anisotropy near the interface. We highlight one issue related to this NEMS application, namely, strain shielding and confined plasticity at the polymer-substrate interface, which leads to undesired rim formation during thermomechanical recording. But first, we begin with a brief review of the glass transition and molecular mobility, followed by an introduction to macromolecular probing techniques involving SPM.

\section{The Glass Transition and Molecular Mobility}

Describing material behavior in terms of transport properties requires, in addition to the structural properties, knowledge of kinetic properties such as molecular mobility. Particularly for condensed, amorphous systems like polymers, accessing molecular mobility is essential. Paramount to any discussion of molecular mobility is the glass transition. By definition, the glass transition is the reversible change in an amorphous material (e.g. polystyrene) or in amorphous regions of a partially crystalline material (e.g. polyethylene), between a viscous or rubbery condition and a hard, relatively brittle one [4]. The temperature at which the transition occurs is defined as the glass transition temperature, $T_{g}$.

The term glass transition is used in the materials community pervasively, implying that it describes a wellunderstood material phenomena. $T_{g}$ is frequently treated as a material property. However, similar to other phenomenological properties, such as friction, a large ambiguity exists. The problem in describing the glass transition, or reporting the transition temperature unambiguously arises from: diverging instrumental observables; critical parameters, such as measurement rates or areas; and sample preparation techniques that generate material anisotropy. The differences arising from these issues has lead to various interpretations and diverging theoretical models. For example, in the discussion that follows, we will differentiate various aspects of thermodynamic versus kinetic approaches to the glass transition.

The definition of the glass transition offered above depends on one's perception of the terms solid and liquid. Materials may be classified as solids and liquids by either considering their rheological response, or by analyzing the thermodynamic phase of the system. A rheological material description is concerned about stress-strain and stress-strainrate relationships. A solid like behavior exhibits a rheological behavior characterized by a purely stress-strain relationship. Conversely, a liquid like behavior is a purely stress versus strain-rate dependent process, which cannot be described with a stress-strain relationship. Any real material will exhibit various degrees of both behaviors, depending on the intrinsic mobility and on the extrinsic stress and stress rate. 
From the thermodynamic perspective of free energy changes between equilibrium states, one may identify the solid-liquid phase transition by a discontinuity in the first partial derivatives of the Gibbs free energy, $G$, with respect to the relevant state variable (e.g., temperature, $T$, and pressure, $P$ ), as illustrated for the volume-temperature plot in Fig. 1.

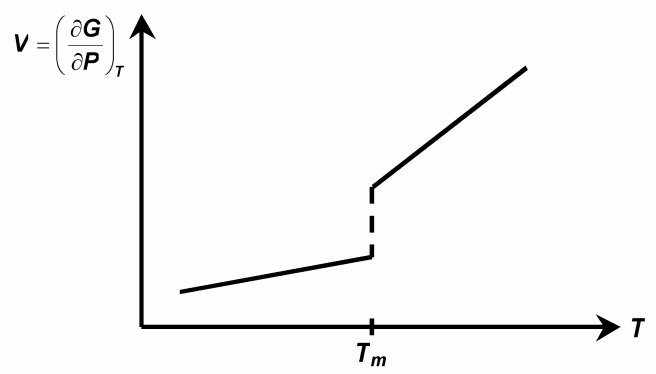

Figure 1. Volume discontinuity at the first order thermodynamic transition between a liquid and solid ( $T_{m}=$ melting temperature).

Discontinuities, as expressed in the first partial derivatives of the Gibbs free energy are found in the temperature relationships of the volume, $V$; entropy, $S$; and enthalpy, $H$, as describe in

$$
\left(\frac{\partial G}{\partial P}\right)_{T}=V ; \quad\left(\frac{\partial G}{\partial T}\right)_{P}=-S ; \quad\left(\frac{\partial(G / T)}{\partial(1 / T)}\right)_{P}=H
$$

Mesophases between solid and liquid phases that are found in polymeric systems include the states of a glass and a melt. The glass state is known to exist also for many nonpolymeric materials. From a structural viewpoint, a solid can be either crystalline, amorphous (unstructured) or partially amorphous-crystalline. A glass is an amorphous solid and can exhibit both solid- or liquid-like behaviors. The melt behaves rheologically liquid-like; yet, exhibits short-range order that is similar to the amorphous solid, but absent in perfect liquids. The melt state compared the corresponding glass-state shows the same structure, but exhibits large amplitude molecular motions, such as translational, rotational, and conformational motions. The large amplitude motions generally operate on the picosecond $\left(10^{-12} \mathrm{~s}\right)$ time-scale. Around the glass transition temperature, the time-scale of this large amplitude motion is slowed to milliseconds or even seconds [5].

Empirically it has been found that for many glasses with mobile units the size of one to six atoms, called beads, the heat capacity increases "abruptly" by about $11 \mathrm{~J}(\mathrm{~mol} \mathrm{~K})^{-1}$ at the glass transition temperature [5]. Discontinuity in the heat capacity is known to exist and can be caused by second-order transitions. Second order transitions are considered as order-disorder transitions, and express a continuous behavior of the free energy and its partial derivatives, and a discontinuous behavior for the second partial derivative with respect to the relevant state variable. Hence there are no discontinuities in $S, V$ or $H$ at the glass transition temperature, but the discontinuities lie in the heat capacity, $C_{p}$, the isothermal compressibility, $\kappa$, and the coefficient of thermal expansion, $\alpha$ :

$$
\begin{array}{ccc}
\mathrm{C}_{\mathrm{p}}: & -\left(\frac{\partial^{2} G}{\partial T^{2}}\right)_{P}=\left(\frac{\partial S}{\partial T}\right)=\frac{C_{p}}{T} \\
& \frac{\partial}{\partial T}\left[\left(\frac{\partial(G / T)}{\partial(1 / T)}\right)_{P}\right]_{P}=\left(\frac{\partial H}{\partial T}\right)_{P}=C_{P} \\
\kappa: & \left(\frac{\partial^{2} G}{\partial P^{2}}\right)_{T}=\left(\frac{\partial V}{\partial P}\right)_{T}=-\kappa V \\
\alpha: & {\left[\frac{\partial}{\partial T}\left(\frac{\partial G}{\partial P}\right)_{T}\right]_{P}=\left(\frac{\partial V}{\partial T}\right)_{P}=\alpha V}
\end{array}
$$

First and second order transitions are illustrated in Fig. 2. If compared to property changes in glasses around the glass transition temperature, one finds some similarity between the glass transition and the second order transition. There are however significant differences. $C_{p}, \kappa$ and $\alpha$ values are always smaller and nearly constant below $T_{g}$ compared to the values above $T_{g}$. This is in contrast to the second-order transition. A more disturbing finding is that $T_{g}$ measurements are highly heating/cooling rate dependent, which does not occur for a "true" second-order transition [6].
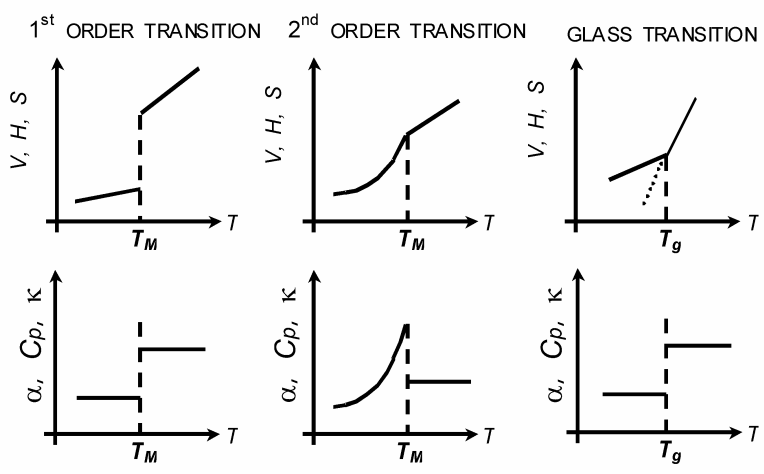

Figure 2. The nature of material property changes at $T_{g}$ compared to those of $1^{\text {st }}$ and $2^{\text {nd }}$ order thermodynamic transitions.

Based on the similarity of the glass transition with a second order thermodynamic transition, the Ehrenfest approach may be applied [6]. Equilibrium criteria requires $G_{\text {glass }}=G_{\text {melt }}$, and the analogous form of the ClausiusClapeyron equation is: 


$$
\frac{d P}{d T_{g}}=\frac{\Delta H}{T \Delta V}
$$

It follows for a second order phase transition

$$
\frac{d T_{g}}{d P}=\frac{\Delta \kappa}{\Delta \alpha}=\frac{T \Delta \alpha}{\Delta C_{p}} \quad \text { or } \quad T_{g}=\left.\frac{\Delta \kappa \Delta C_{p}}{\Delta \alpha^{2}}\right|_{T=T_{g}} .
$$

However for most polymers, the change of $T_{g}$ with pressure has been found to be [7]:

$$
\frac{d T_{g}}{d P}<\frac{\Delta \kappa}{\Delta \alpha} \quad \text { and } \quad \frac{d T_{g}}{d P} \approx \frac{T \Delta \alpha}{\Delta C_{p}}
$$

suggesting that the glass transition is not a true second order thermodynamic transition.

Recognizing the thermal and loading rate dependencies of the glass transition process, the glassy state can be described as a non-equilibrium state. This approach requires, in addition to the state variables, an internal order parameter. In other words, from the concept of an orderdisorder transition, where the glass is the ordered state, the internal order parameter describes the departure from equilibrium conditions. Fox and Flory suggest that the appropriate order parameter is the free volume [6]. The free volume, $V_{f}$, is defined as the void space within the polymer phase that is not occupied by the molecules themselves, and may be quantified by the difference between the total volume, $V$, and the molecular volume, $V_{m}$. The molar volume is the sum of the hypothetical volume in a void free melt at absolute zero and the volume expanded due to thermal vibrations. The relation for the free volume is then:

$$
V_{f}=V+V_{m}\left\lfloor\Delta \alpha\left(T-T_{g}\right)-1\right\rfloor
$$

Just below $T_{g}$, the molecular mobility is so drastically reduced that a non-equilibrium state would become effectively frozen, suggesting a constant free volume below $T_{g}$. From Fig. 2, it is seen that $\Delta \alpha=0$, and with $V$ and $V_{m}$ exhibiting the same temperature dependence, $V_{f}$ becomes constant, see Fig. 3.

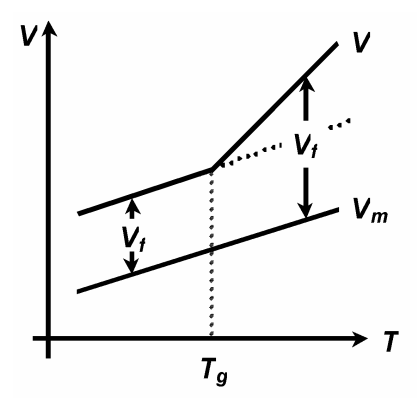

Figure 3. Free volume, $V_{f}$, with respect to temperature.
In the melt state between the glass and liquid states, i.e. for temperatures between $T_{g}$ and the melting temperature, $T_{m}$, the material can be treated as a supercooled fluid. Building from Eyring's model for supercooled liquids [8], the viscosity may be expressed in the form of:

$$
\eta=\eta_{o} \exp \left(\frac{\Delta G_{a}}{k_{b} T}\right) ; \quad \eta_{o}=\frac{k_{b} T}{C_{E} v_{a}}
$$

with $\Delta G_{a}$ as the height of free energy barrier to be crossed by Eyring's jump, the Boltzmann constant $k_{b}$, the activation volume of the stress, $\sigma$, deformation $v_{a}$ (assume $v_{a} \sigma<<k T$ ), and a constant $C_{E}$. With decreasing temperature, i.e. $T \rightarrow T_{g}$, the Arrhenius behavior breaks down, and the viscosity is represented by a power law, or by the empirical Williams-Lendel-Ferry (WLF) relationship [9]:

$$
\log \left(\frac{\eta(T)}{\eta\left(T_{g}\right)}\right)=\frac{-c_{1}\left(T-T_{g}\right)}{c_{2}+T-T_{g}}
$$

where $T \rightarrow T_{g}$ from above; and the constants $c_{1}$ and $c_{2}$ are defined as:

$$
\begin{gathered}
c_{1}=B /\left(2.303 f_{g}\right) \\
c_{2}=f_{g} / \alpha_{f}
\end{gathered}
$$

where $B$ is a constant taken as unity according to Doolittle [6], $f_{g}$ is the fractional free volume, $V_{f} / V$, in the glass transition region, and $\alpha_{f}$ is the coefficient of thermal expansion of the free volume. The apparent activation energy, $E_{A}$, associated with this kinetic model becomes [10]:

$$
E_{A}=-2.303 R \mathrm{c}_{1} c_{2} \frac{T^{2}}{\left(c_{2}+T-T_{R}\right)^{2}}
$$

with $T \geq T_{g}, T_{R}$ is a reference temperature, and $R$ is the gas constant. At $T=T_{g}$, the activation energy takes the form [10]:

$$
E_{A}=-2.303 R\left(c_{1} / c_{2}\right) T_{g}^{2}
$$

Comparing the above theories one finds that the free volume model presumes that the entire motion of atoms results only from the distribution of free volume without crossing the energy barrier. Conversely in the kinetic model, one assumes that the atomic motions are the consequence of a co-operative rearrangement of an assembly of structural units under the effect of thermal fluctuations, which allow the jump over the energy barrier separating the initial and final configurations. It has been very difficult to 
experimentally establish a preference for either of the two theories. We will show in Section 4, that scanning probe microscopy (SPM) measurements in polymer systems favor the kinetic interpretation of the glass transition. However, we will first introduce in the following section, two scanning probe techniques that are especially well suited for the necessary thermorheological studies.

\section{Macromolecular Probing Techniques}

A diverse array of instrumental techniques are employed for glass transition studies. The most classical methods for obtaining $T_{g}$ are calorimetric measurements (differential scanning calorimetry, DSC) that record the specific heat capacity as a function of the temperature, $C_{p}(T)$ [11]. Other methods involve dilatometric measurements for the determination of the specific volume, mechanical property measurements (thermomechanical analysis, TMA, and dynamic mechanical analysis, DMA), and dielectric measurements [4]. Near-edge X-ray absorption fine structure (NEXAFS) spectroscopy[12], X-rays diffraction [13], slow-positron-annihilation spectroscopy (SPAP) [14, 15], brillouin light scattering (BLS) [16, 17], photon correlation spectroscopy and quartz crystal microbalance techniques [18], spectroscopic ellipsometry (SE) [19], attenuated total reflection (ATR) [20], and scanning probe microscopy (SPM) [21-27] have all been employed to determine $T_{g}$ for thin polymer films.

Compared to most spectroscopic and scattering techniques, SPM techniques benefit from the real space molecular sensitivity of a nanoscopic probe. The SPM cantilever tip can be operated in any number of creative approaches for probing nanospace. Two SPM methods that have proved useful in polymer relaxation dynamics and glass transition studies are shear modulated force microscopy (SM-FM) and friction force microscopy (FFM). Both of these techniques are concerned with the application and measurement of lateral forces, and may be classified more generally under lateral force microscopy (LFM). Before we proceed with the experimental details of these techniques, we will first lay a theoretical foundation for the underlying contact mechanics and discuss appropriate LFM calibration methods.

\subsection{Static Contacts}

Contact between two smooth elastic surfaces was first investigated by Hertz, who proposed a simple model, where both the size and shape of contact followed from the elastic properties of the bodies. This model was later extended by two models, Derjaguin-Muller-Toporov (DMT) [28] and Johnson-Kendall-Roberts (JKR) [29], to account also for adhesion. While in the DMT model the adhesion was considered outside the contact, the JKR model restricted the adhesion to the contact zone. Thus, the force acting between the two spheres, composed of both the adhesive and loading forces, is different depending on the model chosen. The "pull-off force", $F_{a d h}$, i.e. the critical negative load, at which two surfaces of combined radii $R$ suddenly break apart, is proportionally related to the work of adhesion $\Delta \gamma$. The proportionality factor is $-1.5 \pi R$ and $-2 \pi R$ in the JKR or DMT limit, respectively.

The apparent discrepancy between the two models was resolved by David Tabor (1977),[30] who suggested a correction parameter, referred to as the Tabor Coefficient $\mu_{T}$, which compares the adhesive interaction strength with the intrinsic deformation properties (i.e., modulus) of the materials. Greenwood and Johnson suggested a Tabor coefficient of the form [31]:

$$
\mu_{T}=\sigma_{o}\left(\frac{R}{E^{* 2} \Delta \gamma}\right)^{\frac{1}{3}} .
$$

where $\sigma_{0}$ is the maximum adhesive stress and the reduced modulus, $E^{*}$, defined as:

$$
E^{*}=\left(\frac{1-v_{1}^{2}}{E_{1}}+\frac{1-v_{2}^{2}}{E_{2}}\right)^{-1},
$$

where $E_{i}$ and $v_{i}$ are the corresponding Young's modulus and the Poisson ratio, respectively. A similar relationship was introduced by Maugis [32] with:

$$
\lambda=2\left(\frac{16}{9 \pi}\right)^{1 / 3} \sigma_{\circ}^{*}\left(\frac{R}{E^{* 2} \Delta \gamma}\right)^{\frac{1}{3}},
$$

assuming a constant adhesive stress $\sigma_{0}{ }^{*}$ represented by a square well ("Dugdale") interaction potential. Barthel, and Greenwood and Johnson showed that Maugis' "Dugdal approximation" is also applicable to more general potentials [33], and to predict load-separation curves and pull-off forces that are adequate. It could be shown that if $\mu_{T}$ or $\lambda$ exceed 5, the JKR model applies, i.e. tensile and compressive surface forces have to be considered, and for $\mu_{T}$ or $\lambda$ smaller than 0.1 the DMT model is applicable.

Based on Maugis' analytical solution to adhesive contact problems, Carpick et al. developed a "generalized equation" for the normalized contact area between the contact limits of the JKR and DMT models, using a single fitting parameter $\alpha$, which is related to Maugis' elasticity parameter as follows [34]:

$$
\lambda=-0.924 \times \ln (1-1.02 \alpha) \text {. }
$$


The fitting parameter $\alpha$ is given by the variation of the contact radius $a$ with the ratio of the applied load, $F_{a p p}$, and the adhesive force, $F_{a d h}$, by [34]:

$$
\frac{a}{a_{o(\alpha)}}=\left(\frac{\alpha+\sqrt{1+F_{a p p} / F_{a d h(\alpha)}}}{1+\alpha}\right)^{\frac{2}{3}}
$$

Here, the contact area at zero load, $a_{o}$, and the $F_{\text {adh }}$ are dependent on $\alpha$. For $\alpha=0$ and $\alpha=1$, Eq. 15 provides the normalized contact radius in the DMT and JKR limit, respectively, with a contact radius at zero load of:

$$
a_{o}^{3}=\frac{3}{4} \frac{R}{E^{*}}(6 \pi \gamma R)=\frac{9}{2} \frac{\pi \gamma R^{2}}{E^{*}}(\mathrm{JKR})
$$

and

$$
a_{o}^{3}=\frac{3}{4} \frac{R}{E^{*}}(2 \pi \gamma R)=\frac{3}{2} \frac{\pi \gamma R^{2}}{E^{*}}(\mathrm{DMT}) .
$$

\subsection{Modulated Contacts}

Now, let us turn our attention to SPM type contacts that involve sinusoidal modulations in either the normal $(z)$ or lateral $(x)$ direction. The contact stiffness experienced during normal modulation experiments is typically defined as the gradient of the equilibrium force between tip and sample, and is given as [35]:

$$
k_{c, z}=2 a E^{*}
$$

The normal contact stiffness $k_{c, z}$ is experimentally obtainable from normal displacement measurements, which are distributed between two springs in series; i.e.:

$$
\Delta z=\Delta z_{c}+\Delta z_{N}
$$

where $\Delta z_{C}$ and $\Delta z_{N}$ correspond to the elastic deformation of the sample (or more generally the contact) and the elastic normal cantilever displacement, respectively. The elastic constant, $k_{\text {tot, } z}$, corresponding to the total normal deformation $\Delta z$ is thus related to the sample contact stiffness $k_{c, z}$ and the normal cantilever stiffness $k_{N}$, as:

\footnotetext{
* Note that for a Hertzian contact the radius is given by: $a^{3}=\frac{3}{4} \frac{R}{E^{*}} F_{L}$.
}

$$
\frac{1}{k_{t o t, z}}=\frac{1}{k_{c, z}}+\frac{1}{k_{N}} .
$$

For small in-plane lateral displacements in $x$, similar equations can be derived under the assumption of no-slip. For a sphere-plane geometry, the lateral stiffness of the contact $k_{c, x}$ is provided by Mindlin's theory $[36,37]$ as:

$$
k_{c, x}=8 a G^{*}
$$

with

$$
G^{*}=\left(\frac{2-v_{1}^{2}}{G_{1}}+\frac{2-v_{2}^{2}}{G_{2}}\right)^{-1}
$$

where $G_{1}$ and $G_{2}$ are the shear moduli of the sample and the probing tip, respectively, and $v_{1}$ and $v_{2}$ are the corresponding Poisson's ratios [38].

In analogy to the normal deformation, we introduce the total lateral elastic constant as:

$$
\frac{1}{k_{t o t, x}}=\frac{1}{k_{c, x}}+\frac{1}{k_{T}},
$$

where $k_{c, x}$ and $k_{T}$ stand for the lateral contact stiffness and the torsional cantilever stiffness, respectively. As for normal deformations, it is generally assumed that the shear deformation of the cantilever is restricted to the bending of the bar, and thus, any tip deformations are neglected. This assumption is appropriate for soft organic materials; however, may breakdown when contacting hard samples like silicon wafers.

For force modulation measurements, the modulation load, $F_{\text {mod }}$, is varied around the equilibrium load, $F_{o}$, with an oscillating perturbation, often in the form of a sine wave. The modulation signal is generally provided by a function generator, and may be applied to either the cantilever or the sample. A first order approximation for the overall force in the modulation direction, $i$, provides:

$$
F_{i}=F_{o}+\frac{\partial F_{m o d}}{\partial x_{i}} \Delta x_{i}
$$

where

$$
\frac{\partial F_{m o d}}{\partial x_{i}}=k_{t o t, i}
$$

and $i=z$ or $x$ for normal or lateral modulation, respectively, and $\Delta x_{i}$ represents the corresponding input modulation amplitude. The equilibrium load $F_{o}$ represents the combination of the applied and adhesive loads for normal force modulation, $F_{a p p}$ and $F_{a d h}$, respectively. In shear 
modulation, $F_{o}$ is either zero under no-slip conditions, or corresponds to the friction force, $F_{F}$, in a sliding contact.

\subsection{Calibration of Lateral Forces in Scanning Probe Microscopy}

Lateral forces are still relatively difficult to quantify accurately. Due to the geometric uncertainties in SPM probes, mathematical calibration methods are often inaccurate. Hence, at least two experimental calibration techniques have been offered: a lateral contact stiffness calibration [37], and a blind friction calibration [39]. Both methods assume that the normal force, $F_{N}$, and hence the normal cantilever stiffness, $k_{N}$, are known. The normal force is the sum of the applied and adhesive loads, i.e. $F_{N}=F_{a p p}$ $+F_{a d h}$, where the applied load is determined by:

$$
F_{a p p}=k_{N} \frac{\Delta I_{N}-I_{N}^{O}}{S}
$$

where $k_{N}$ is the normal lever stiffness, $S$ is the sensitivity of the detection scheme (generally a photodiode), $\Delta I_{N}$ is the normal deflection signal (i.e. the setpoint value in feedback control), and $I_{N}{ }^{\circ}$ is the initial, out-of contact, normal deflection signal. The sensitivity and adhesion force are determined from force-displacement (FD) curves. Note, that for studies on compliant materials, the adhesion force determined from FD curves is generally convoluted with the contact area. To avoid load dependent changes in the contact area, the adhesion force should be determined from friction versus load measurements, e.g. see Fig. 7.

With commercially available cantilevers, a range for $k_{N}$ is specified by the manufacturer. Due to limitations in the production process, the specified range for $k_{N}$ may span one order of magnitude. The prudent microscopist often seeks a more accurate calibration. For bar shaped cantilevers, the normal and torsional stiffness may be calculated based on the lever geometry, i.e:

$$
\begin{aligned}
& k_{N}=\frac{E W T_{L}^{3}}{4 L^{3}} \\
& k_{T}=\frac{G W T_{L}^{3}}{3 L H^{2}}
\end{aligned}
$$

where $E$ and $G$ are the Young's and shear moduli, respectively. The geometric parameters $W, T_{L}$, and $L$ are the width, thickness, and length of the cantilever, respectively; and $H$ it the height of the probing tip at the end of the lever. Any uncertainty in $T_{L}$ is propagated in Eq. 27 with a power of three; hence, an accurate value for $T_{L}$ is necessary. Again, manufacturer specifications are usually broad. The first resonance frequency in the normal direction, measured via spectrum analysis, can be used to determine $T_{L}$ more accurately [35]:

$$
T_{L}=\frac{2 \sqrt{12} \pi}{(1.875104)^{2}} \sqrt{\frac{\rho}{E}} v_{1} L^{2}
$$

For silicon cantilevers the density $\rho$ is $2.33 \times 10^{3} \mathrm{~kg} \mathrm{~m}^{-3}$ and the modulus $E$ is $1.69 \times 10^{11} \mathrm{~N} \mathrm{~m}^{-2}$ (reference [35]), and Eq. 28 becomes $T_{L}(\mathrm{~m})=7.23 \times 10^{-4}\left(\mathrm{~s} \mathrm{~m}^{-1}\right) \cdot v_{1}\left(\mathrm{~s}^{-1}\right) \cdot L(\mathrm{~m})^{2}$. Alternatively, the normal spring constant for a lever of any geometry may calibrated by measurement of the thermally activated power consumption [40], or by the addition of known masses (styrene spheres) [41].

Once the cantilever is calibrated for normal forces, the next step is the lateral force calibration. The lateral contact stiffness approach is based on SM-FM operation (Fig. 4), which uses lock-in detection to relate the lateral displacement (modulation input, $\Delta x_{i}$ ) with the lateral force signal (amplitude response, $\Delta x_{R}$ ). On any sample, a plot of $\Delta x_{R}$ versus $\Delta x_{i}$ exhibits two regimes, a static region where $\Delta x_{R}$ increases linearly with $\Delta x_{i}$, and a sliding region where $\Delta x_{R}$ saturates at the friction force. The slope of the static region is a direct measure of the overall lateral contact stiffness, related with the calibration factor $C$, i.e.:

$$
k_{\text {tot }, x}=\left.C \frac{d\left(\Delta x_{R}\right)}{d\left(\Delta x_{i}\right)}\right|_{\text {static }}=C k_{\text {tot }, x}^{*}
$$

Substituting equations 21 and 29 into Eq. 23 gives:

$$
\frac{1}{k_{\text {tot }, x}^{*}}=\frac{C}{8 G^{*} a}+\frac{C}{k_{T}}
$$

For a bar shaped cantilever, equations $27 \mathrm{a}$ and $27 \mathrm{~b}$ may be used with $G=E[2(1+v)]^{-1}$ to determine the torsional cantilever stiffness, i.e.:

$$
\frac{k_{T}}{k_{N}}=\frac{2}{3(1+v)}\left(\frac{L}{H}\right)^{2}
$$

Alternatively, the torsional stiffness may be obtained by using the Sader [42] and Neumeister [43] formulas. The difficulty with Eq. 30 lies in the selecting the appropriate model for the contact radius, a. Assuming Hertzian contact, Piétrement et al. [37] have shown that this procedure works well for silicon and mica. The Hertzian (or DMT) contact radius is:

$$
a=\left(\frac{3 R}{4 E^{*}}\right)^{1 / 3} F_{N}^{1 / 3}
$$


where $\mathrm{R}$ is the radius of curvature of the SPM tip, and Eq. 30 becomes:

$$
\begin{aligned}
& \frac{1}{k_{t o t, x}^{*}}=\frac{C}{\beta F_{N}^{1 / 3}}+\frac{C}{k_{T}} \\
& \beta=8 G^{*}\left(\frac{3 R}{4 E^{*}}\right)^{1 / 3}
\end{aligned}
$$

where

A linear fit to the experimentally measured $1 / k_{\text {tot }, x}^{*}$ versus $F_{N}^{-1 / 3}$ gives an intercept, $b$, which from Eq. $33, C=b k_{T}$. Finally, the force associated with a lateral displacement of $\Delta x_{i}$ is $F_{L}=C \Delta x_{R}$.

This calibration method becomes cumbersome when more sophisticated models are used for the contact radius in Eq. 30. For example, Carpick's [34] general contact area equation (Eq. 15) requires knowledge of the lateral (friction) force, as well as the tip radius of curvature, to determine the fit parameter $\alpha$. Numerical methods would be required to iteratively fit the contact radius and the calibration curves. The blind friction calibration is model independent and avoids these complications by directly measuring a calibration factor on a well characterized, commercially available silicon standard, that has a prescribed friction coefficient, $\mu_{S i-S i}$. The trade-off however, is the possibility of surface contamination on the silicon standard, which would alter the actual friction coefficient. To avoid this, careful silicon treatment is necessary [39] From Amonton's Law, it is known that

$$
F_{L}=\mu_{S i-S i} \cdot F_{N}
$$

Using a well defined cantilever that was characterized by scanning electron microscopy (SEM), Buenviaje et al. determined a value of $\mu_{S i-S i}=0.18 \pm 0.03$ [39]. The lateral forces experienced by the cantilever tip are directly proportional to the lateral deflection signal of the SPM detection system, i.e.

$$
F_{L}=\Gamma \cdot \Delta I_{L}
$$

where the proportionality constant $\Gamma$ is the calibration factor being sought, and $\Delta I_{L}$ is the lateral deflection signal. $\Delta I_{L}$ is measured on a well treated silicon oxide surface for a range of normal forces, $F_{N}$. For positive applied loads $\left(F_{a p p}>0\right)$, a linear fit to the measured $\Delta I_{L}\left(F_{N}\right)$ gives the calibration curve

$$
\Delta I_{L}=m_{\text {cal }} \cdot F_{N}
$$

Dividing equations 34 and 36 yields:

$$
F_{L}=\frac{\mu_{S i-S i}}{m_{\text {cal }}} \cdot \Delta I_{L}
$$

Thus the calibration factor $\Gamma$ in Eq. 35 becomes:

$$
\Gamma=\frac{\mu_{S i-S i}}{m_{c a l}}=\frac{0.18 \pm 0.03}{m_{c a l}}
$$

With a value for $\Gamma$, Eq. 35 may be used to quantify the lateral forces measured by the SPM detection scheme.

The silicon treatment is necessary to generate a clean and stable silicon oxide surface. This is not to be confused with stripping or hydrogen passivation techniques, e.g. HF treatment, which remove the surface oxide layer and render an unstable surface. The procedure of Buenviaje et al. [39] requires sequential sonication of the silicon calibration sample; first in acetone for 15 minutes, then in methanol for 30 minutes (HPLC grade from commercial sources). The sonication steps are followed by rinsing with ultra-pure water. Finally, the silicon calibration standard is heated above $100{ }^{\circ} \mathrm{C}$ in a low humidity environment, preferably a vacuum oven, to remove excess water. This procedure provides a reproducible calibration sample for up to two hours, depending on the post-treatment environmental conditions, i.e. relative humidity. With a calibrated SPM system, one is prepared for experimental endeavors based on the following lateral force probing techniques.

\subsection{Shear Modulation Force Microscopy (SM-FM)}

Characterization efforts using SPM modulation techniques seek to infer material specific information from the elastic (or dissipative) nature of the probing contact. Shear modulation force microscopy (SM-FM) is one approach that is especially well suited for surface rheological studies. SM-FM has proven to be particularly successful in determining crosslinking densities and structural phase transitions in polymeric systems [26, 27]. The working principle of SM-FM is sketched in Fig. 4.

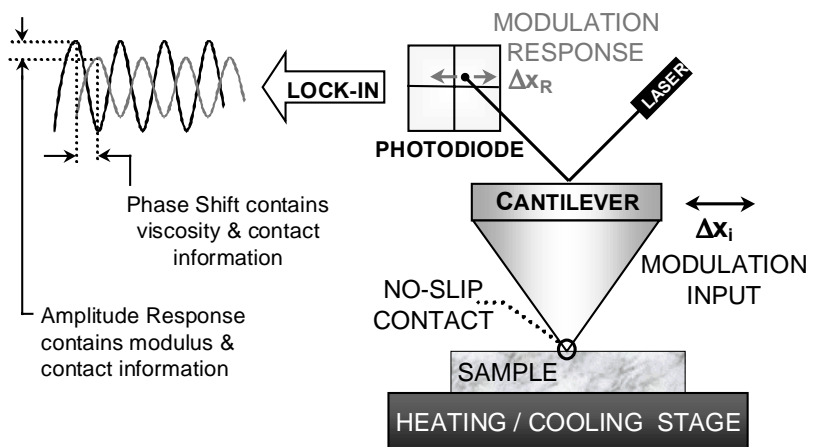

Figure 4: Working Principle of Shear Modulated Force Microscopy (SM-FM).

It involves a nanometer sharp cantilever tip that is maintained in contact with a sample surface, under a constant load of roughly $(5-100) \mathrm{nN}$. The tip is laterally 
modulated with a nanometer amplitude, $\Delta x_{i}$, that avoids any tip-sample slipping. Using lock-in techniques, the modulation response, $\Delta x_{R}$, is analyzed relative to $\Delta x_{i}$. The response amplitude is a measure of the total elastic constant, $k_{\text {tot }, x}$, and the intrinsic sample behavior is captured through the relations in Eqns. 21 and 23. Characteristic of most SPM techniques, two experimental difficulties arise: the accurate determination of the true contact area (radius $a$ in Eq. 21), and accurate evaluation of the cantilever stiffness, $k_{T}$ in Eq. 23. This raises the important point of calibration methods (discussed above). However, in simple thermorheological studies like glass transition temperature measurements, the complications associated with a poorly defined contact area and lever stiffness are inconsequential.

For thermal analyses, the sample temperature is increased stepwise by $(0.5-2.0){ }^{\circ} \mathrm{C}$ increments. At each temperature, thermal equilibrium is obtained before any viscoelastic responses are recorded. The response amplitude, $\Delta x_{R}$, is plotted versus temperature. For glass transition measurements, the $T_{g}$ is determined from the "kink" in the response curve, as reported in Fig. 5(a).
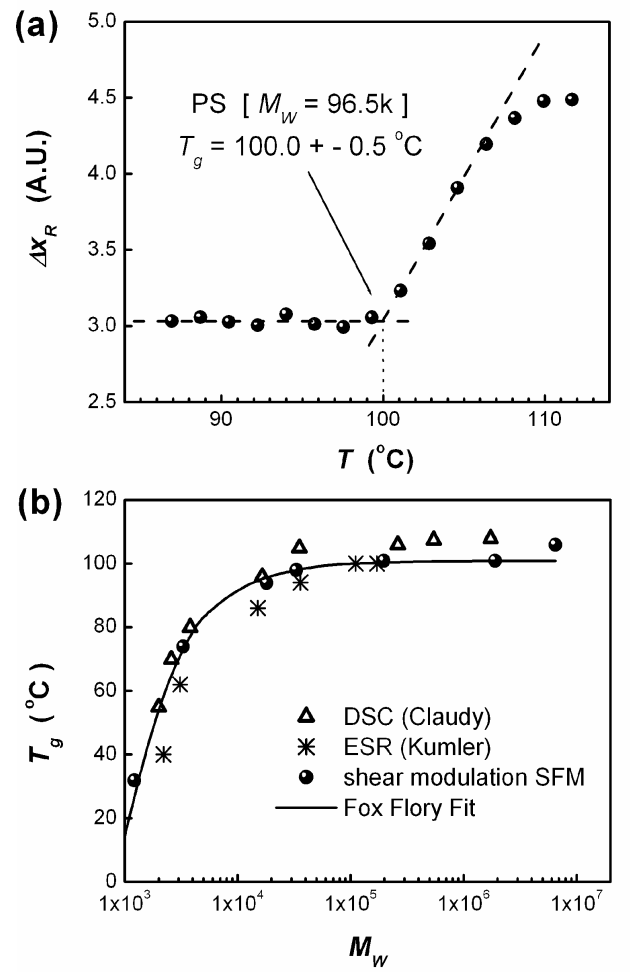

Figure 5: SM-FM glass transition temperature, $T_{g}$, measurements. (a) The modulation amplitude response indicating the $T_{g}$ of polystyrene. (b) Comparison of SM-FM $T_{g}$ values as a function of molecular weight against other methods and theory Date for (b) from reference [46].

Below $T_{g}$, the probing depth of SM-FM is on the order of $1 \mathrm{~nm}$, which allows substrate-independent measurements down to film thicknesses of a few nanometers. Any surface effects less than $1 \mathrm{~nm}$ in depth [44] cannot be addressed under these conditions. The slow creeping process above $T_{g}$ is documented elsewhere [45]. While the accuracy of SM-FM $T_{g}$ measurements compares well with other techniques [46], Fig. 5(b), SM-FM also offers the versatility for probing rheological properties in confined sample geometries.

It is important to note that the SM-FM technique described here is a non-scanning method. The reason is briefly describe: To obtain high accuracy in $T_{g}$ measurements it is essential not to induce, by other means than temperature, changes in the contact area. This is to avoid system-driven artifacts in the contact stiffness, $k_{c, x}$. To be precise, $k_{c, x}\left(A_{L}, G^{*}\right)$ in Eq. 21 , i.e. the resistance of the contact to deform, is dependent on (a) the laterally projected contact area, $A_{L}$, (e.g. the side wall of an indentation tip), and (b) the relative shear properties of the two materials, $G^{*}$. Thus, any local plastic deformation, for instance, the generation of a deformation wave (Schallamach wave) [47] that travels ahead of a scanning SFM tip can change $k_{c, x}$. Plastic deformation is intrinsically rate and load dependent. Therefore, it is not astonishing that scanning methods, such as the friction force microscopy, have revealed scan rate dependent apparent $T_{g}$ values [25]. By placing the SFM tip stationary at constant load onto the polymer surface, contact area changes occur only due to temperature induced changes in the sample modulus, i.e. there are no load induced changes in the contact radius, $a$, and the cantilever stiffness, $k_{L}$, is essentially constant. Consequently, the experimental observable in the SM-FM method, $k_{t o t, x}$, is changing only due to changes in the polymer material properties. With this, the "kink", observed in Fig. 5(a) is a true measure of the transition property.

\subsection{Friction Force Microscopy (FFM)}

The advantages to the non-scanning SM-FM technique were highlighted above. Now, let us discuss the complimentary advantages to the scanning approach of friction force microscopy (FFM). In fact, both methods may be superimposed and conducted simultaneously, if the timescales for the SPM feedback system, modulation, and scanning do not overlap. Nevertheless, we will restrict ourselves here to the principles of FFM and discuss the inherent tribological attributes of this method.

Friction force microscopy simulates a single asperity provided by an ultra-sharp tip on a soft cantilever, pictured in Fig. 6. The small contact area, on the order of the molecular dimensions, is insufficient to confine macromolecules and generally allows discussing friction results in terms of thermodynamic equilibrium. Hence, FFM is not appropriate to reflect on tribological issues involving large area confinement effects. FFM offers sufficient sensitivity for friction measurements on the molecular scale, and has been used to detect molecular stick-slip behavior [48]. Although FFM images are valuable for assessing surface heterogeneity and the decomposition of blends, investigations of macromolecular dynamics require a 
quantitative friction analysis. The ability to conduct thermal, rate, and load dependent friction studies makes FFM especially well suited for investigating the time-temperature nature of viscoelastic materials.
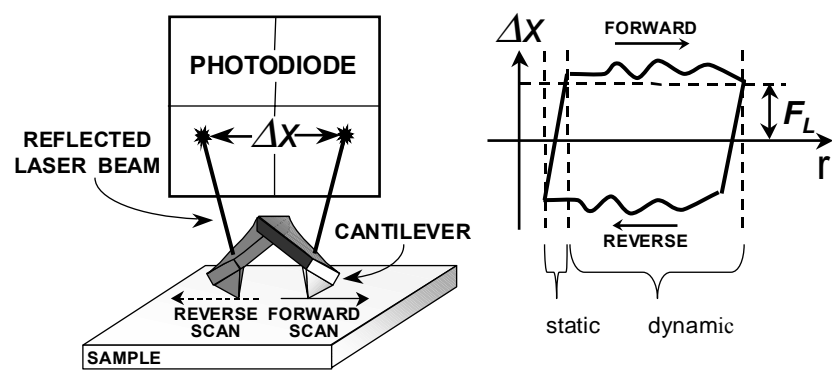

Figure 6. Operating Principle of Friction Force Microscopy (FFM).

The friction force, $F_{F}$, is measured through the torsional bending of the cantilever during scanning, pictured in Fig. 6 . The hysterisis in the lateral displacement signal, between forward and reverse scans, is proportional to the energy dissipated through friction. Absolute values for the friction force are determined by first calibrating the cantilever and detection scheme with one of the methods described in Section 3.3. Its important to note that any changes to the laser alignment will invalidate the calibration. Further, it is important that friction analyses on compliant materials are adhesion corrected, shown in Fig. 7.

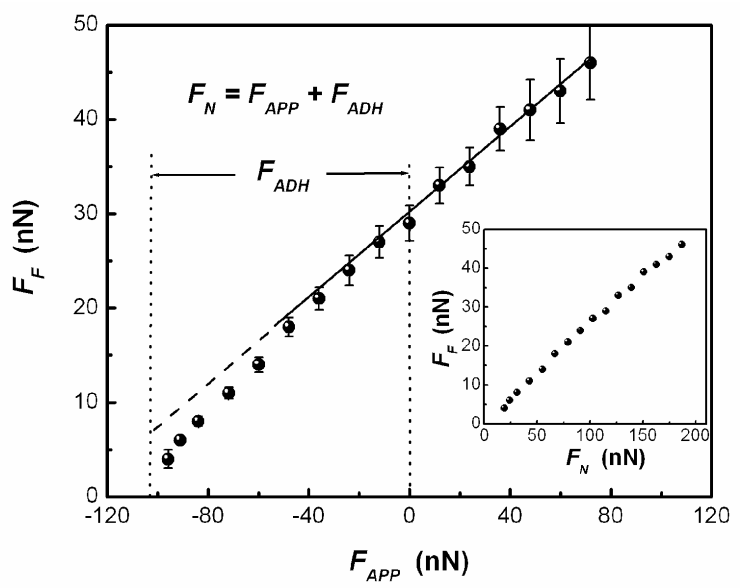

Figure 7. Adhesion correction of friction force measurements on poly(methyl methacrylate) (PMMA).

This is necessary to account for load dependent changes in the contact area, i.e. $F_{F}$ must be described in terms of the total normal force which is the sum of the applied load, $F_{\text {app }}$, and the adhesion force, $F_{a d h}$.

\subsection{Tribological Models for FFM}

The frictional resistance to sliding of the FFM tip is generally discussed in terms of a thermal activation model, the Eyring model [8], which employs a regular series of potential barriers that are continuously overcome during the sliding process. Briscoe et al [49] applied this idea to interpret the frictional behavior observed on molecularly smooth, soap-like lubricants, and derived the following shear strength versus velocity $v$ relationship [49]:

$$
\tau=\frac{k_{B} T}{\phi} \ln \left(\frac{v}{v_{o}}\right)+\frac{1}{\phi}(Q+P \Omega) .
$$

The barrier height, $E$, is composed of the process activation energy $Q$; the compression energy $P \Omega$, where $P$ is the pressure acting on the volume of the junction $\Omega$; and the shear energy $\tau \phi$, where $\tau$ is the shear strength acting on the stress activation volume $\phi$. $T$ represents the absolute temperature, and $v_{o}$, is a characteristic velocity related to the frequency of the process and to a jump distance. The stress activation volume $\phi$ can be conceived as a process coherence volume, and interpreted as the size of the moving segment in the unit shear process, whether it is a part of a molecule or a dislocation line. Thus, Eyring's model predicts a linear relationship of friction (the product of the shear strength and the active process area) in pressure and temperature, and a logarithmic relationship in velocity.

The Eyring model has been verified in experiments on solid lubricants of an inherent, highly ordered structure [49] and also for a liquid system where a series of potentials is built up and overcome in the course of the shear process [50]. Furthermore, Gnecco et al [51] showed in an ultrahigh vacuum FFM study on sodium chloride, that the concept of the Eyring model also applies for dry friction on an ordered surface. Thus, a molecular theory of friction could be derived from a very simplistic model of an apparent sinusoidal-corrugated surface potential over which a cantilever tip is pulled. However, such a simple model assumes there is no noise present, such as thermal noise, and thus, the driven tip leaves the potential well when the barrier vanishes at the instability point. This leads to recent theoretical treatments that consider barrier-hopping fluctuations associated with thermal noise, i.e. creep models [52-54]. With creep, the FFM tip slips to the next potential well at lower energy values than those prescribed by the barrier height.

Early considerations of thermal fluctuations by Heslot et al. [54] (1994) led to a friction force that is logarithmically dependent on the velocity, $v$, similar to the Eyring model:

$$
F_{F}=\text { const }+\ln (v)
$$

In Heslot's linear creep model, the barrier height is proportional to the frictional force. Sang et al. [53] argued 
that with an absorbing boundary condition, i.e. an elastic deformation of the overall potential due to the driven motion of the cantilever, the barrier height becomes proportional to a $3 / 2$ power law in the friction force. Sang's modifications resemble a ramped creep model, and lead analytically to the following friction-versus-velocity relationship:

$$
F_{F}=F_{c}-\Delta F_{F}\left|\ln v^{*}\right|^{2 / 3}
$$

where $v^{*}$ represents a dimensionless velocity, $\Delta F_{F} \propto T^{2 / 3}$, and $F_{c}$ is an experimentally determined constant [53]. The same relationship of friction with velocity was also derived for the maximum spring force by Dudko et al [52].

Although there is currently no experimental data available that would provide friction force measurements over a sufficiently large temperature and velocity range to verify the ramped creep model, the stronger supporting argument for ramped creep is its comparison to the numerical solution of the Langevin equation. The Langevin equation combines the equation of motion (including the corrugated surface potential and perfect cantilever oscillator in the total potential energy, $E$ ) with a random force, $\xi(t)$, to account for the thermal noise, i.e.:

$$
M \ddot{x}+M \beta \dot{x}+\frac{\partial E(x, t)}{\partial x}=\xi(t)
$$

where $E(x, t)=\frac{k}{2}(R(t)-x)^{2}-U_{o} \cos \left(\frac{2 \pi x}{\lambda}\right)$.

$x$ is the position of the cantilever stage, $\beta$ is the microscopic friction coefficient or dissipation (damping) factor, $\lambda$ is the lattice constant, $U_{o}$ is the surface potential barrier height, $M$ and $k$ represent the mass and the spring constant of the cantilever, respectively. Equation 42a was solved numerically by both groups, Sang et al [53] and Dudko et al. [52], assuming a Gaussian fluctuation-dissipation relation for the random force, i.e., $\left\langle\xi(t) \xi\left(t^{\prime}\right)\right\rangle=2 M \beta k_{b} T \delta\left(t-t^{\prime}\right)$ where $\delta\left(t-t^{\prime}\right)$ is a Dirac function. Sang confirmed the ramped creep model and Dudko showed that a force reconstruction approach from the density of states, accumulated from the corresponding Fokker-Planck equation, is equivalent with the Langevin equation.

At this point, let us consider the possibility that hindered, or frozen, relaxation states of an amorphous polymer could be activated in the course of a frictional sliding process, and thus, give rise to barrier-hopping fluctuations not unlike the ones discussed above for highly ordered surfaces. Isothermal friction-velocity curves for glassy polystyrene are presented in Fig. 8. Quasilogarithmic friction-velocity relationships are found, which agree with the above theoretical models [49, 52-54], and resemble FFM experiments on ionic crystals in ultrahigh vacuum [51] and in lubricated sliding [50].

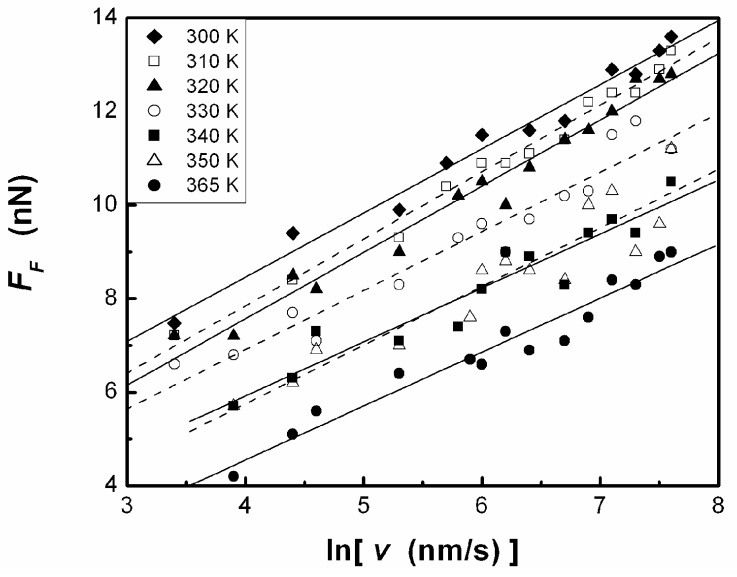

Figure 8. Friction force - scan velocity isotherms for FFM measurements on glassy polystyrene $\left(M_{W}=96.5 \mathrm{k}, M_{W} / M_{N}=1.04, T_{g}=373 \mathrm{~K}, F_{N}=15 \pm 2 n N\right)$.

The friction measurements in Fig. 8 are scaled by the creep models (equations 40 and 41) in Figures 9(a) and 9(b). The ramped creep model in Fig. 9(a), provides a marginally better fit than the linear creep model, Fig. 9(b). The fit quality of the ramped versus linear creep model could be expected to increase for a system that is less overdamped, i.e. a stiffer spring with respect to $\beta$ [55], and with measurements over larger velocity and temperature ranges.

The potential barrier which is continuously overcome during sliding across glassy polystyrene is associated with the hindered rotation of the phenyl side-chains, $7 \mathrm{kcal} \mathrm{mol}^{-1}$ [56]. Evaluation of the barrier height is discussed in Section 4. Considering that the creep models (a) fit the friction data reasonably well, and (b) are in accordance with a fluctuation model based on a Gaussian fluctuation distribution, it appears that there is little or no correlation between the individual phenyl rotations that are relaxed during the shear process. It is because of this weak correlation that similar frictional dissipative behaviors are observed on both highly structured (crystalline) surfaces and the unstructured (amorphous) glassy PS.

The FFM results and associated discussion of molecular friction on a glassy polymer illustrate the effectiveness of rudimentary non-equilibrium models that incorporate simple potentials with thermal fluctuations. Deviations from the models are expected, particularly for correlated fluctuations, i.e. memory effects in relaxation processes. Addressing the issue of the so called non-Markovian behavior of sliding systems demands a more rigorous theoretical treatment, such as the generalized Fokker-Planck equation with a system specific, statistical kernel [57-60].

The discussion above is founded strictly on tribological principles; however, the experimentally determined potential barrier for the PS example is attributed to a rheological relaxation process. One would expect then, that friction could also originate from other relaxations. For example, Hammerschmidt et al. have identified the 
$\beta$-relaxation as the primary dissipation mechanism in FFM measurements on poly(methyl methacrylate) [23].
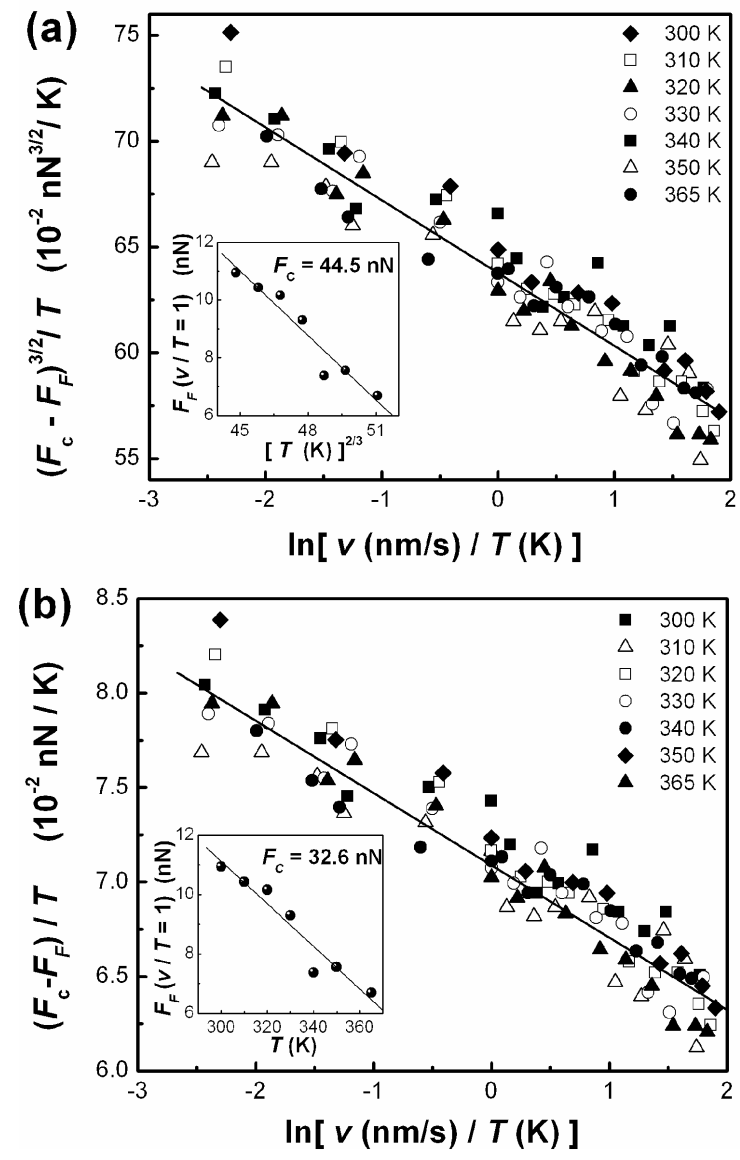

Figure 9. Collapse of the friction data from Fig. 8 to creeping friction models: (a) Ramped creep [53] (regression $\mathrm{R}^{2}=0.9124$ ). Inset: $F_{c}$ is determined from the intercept of $F_{F}$ versus $T^{2 / 3}$ for a fixed ratio $T / V=1 \mathrm{~K} /(\mathrm{nm} / \mathrm{s})$ [53]: $F_{c}=44.5 \mathrm{nN}$. (b) Linear creep [54] (regression $\mathrm{R}^{2}=0.909$ ). Inset: $F_{c}$ is determined from the intercept of $F_{F}$ versus $T$ for a fixed ratio $T / v=1 \mathrm{~K} /(\mathrm{nm} / \mathrm{s})[53]: F_{c}=32.6 \mathrm{nN}$.

The question of to what extent the intrinsic rheological properties are coupled with external tribological attributes in FFM measurements has not been answered unambiguously. This results, in part, from varied experimental conditions, as well as, inappropriate assumptions during the data analysis. Operating the FFM tip at conditions which induce wear or other plastic deformation processes will produce external tribological attributes. This is sometimes difficult to avoid. The relative humidity is another key parameter which effects FFM measurements. For a relative humidity greater than roughly $30 \%$, a capillary neck is formed at the FFM tip due to physisorbed water [61]. In this scenario, the FFM measurements reflect the behavior of a two phase system. In the following section, we attempt to move past some of these issues, and address the utility of FFM for inferring intrinsic rheological characteristics of polymers.

\section{Internal Friction and Dynamics Near the Glass Transition}

A nanoscopic description of polymer dynamics involves, in general, only two parameters: an internal, or monomeric, friction coefficient and an appropriate macromolecular length scale [62]. Monomeric friction dictates the degree of local segmental motion, and thus, is responsible for the bulk viscoelastic properties of polymers. The macromolecular length scale provides a measure for the range of energy transfer, for instance, the size of cooperatively rearranging regions (CRRs) in the heterogeneous dynamics of glass formers. The measurement of nanoscopic critical lengths warrants a local probing technique, such as SPM. This point is illustrated below with molecular insight into the energy dissipation processes in amorphous polystyrene (PS), a material technologically relevant to photonics [63], electronics [64], and nanoelectromechanical systems (NEMS) [3]. We show how both the energetics involved in frictional dissipation and the length scale over which the energy is dissipated can be directly linked to the molecular relaxation and clustering processes that evolve during the glass transition.

\subsection{Molecular Relaxations}

The origin for frictional dissipation in elastomeric materials has been in question for nearly half of a century. Since Grosch [65] (1963) and Ludema and Tabor [66] (1966), a molecular length scale has been accessible in friction experiments involving elastomers in sliding contact with hard surfaces. However, because of the macroscopic nature of these early investigations, segmental chain slippage could only be suspected as the basic mechanism for sliding dissipation [65, 66]. Early interpretations by Schallamach [67] and Ludema and Tabor addressed the friction related segmental motions of the polymer chain from two different viewpoints: Schallamach considered the friction force in terms of a fully adhesive, rate-dependent molecular debonding model, introduced by Frenkel [68] and Eyring [8] and later improved by Chernyak and Leonov [69]. Ludema and Tabor, on the other hand, suggested an adhesive viscoelastic model in which the energy dissipated during sliding is lost in deformation of the soft material. In recent work involving smooth macroscopic sliding contacts, the importance of both processes during steady sliding was recognized $[70,71]$.

Recent FFM studies have benefited from the molecular sensitivity of the SPM probe. A first glance of frictional behavior on polystyrene near its glass transition is illustrated with the friction coefficient, $\mu$, in Fig. 10. The frictional dissipation mechanism shifts from side chain relaxation in the glass phase to backbone relaxation in the melt phase. The transition is not abrupt, but ranges over $15 \mathrm{~K}$, starting at the glass transition temperature $\left(T_{g}=373 \pm 1 \mathrm{~K}\right)$. 


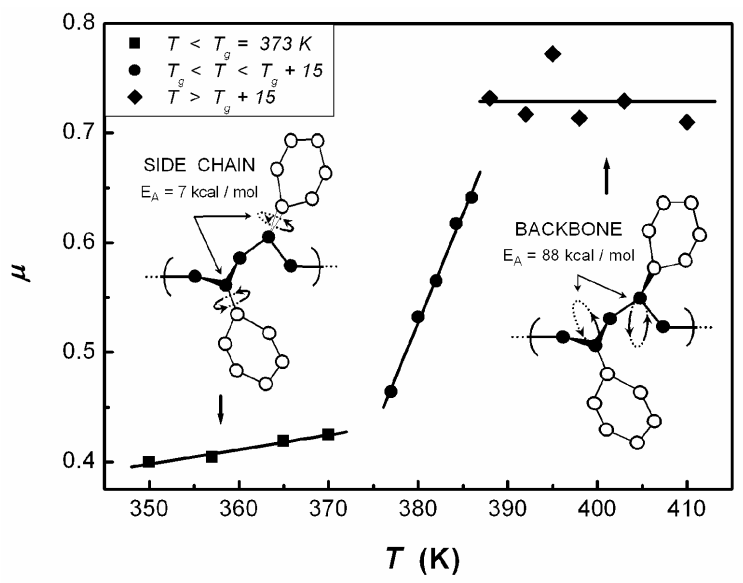

Figure 10. Friction coefficient, $\mu$, and corresponding molecular dissipation mechanisms for atactic polystyrene $\left(M_{w}=96.5 \mathrm{k}, T_{g}=373 \mathrm{~K}\right)$.

The dissipation mechanisms in Fig. 10 were determined from independent FFM friction-velocity analyses, below and above $T_{g}$. An energetic analysis of FFM results begins with the superposition of friction-velocity isotherms, $\left.F_{F}(v)\right|_{T}$, using the method of reduced variables [10], Fig. 11. This linear approach serves to decouple the thermal and rate contributions to the friction force. While the thermal behavior is captured in the horizontal $a_{T}$ shift function, the rate behavior is portrayed in the resulting superposed friction master curve. The mathematical mechanics of the superposition are pictured in Fig. 12.

Below $T_{g}$, the Arrhenius behavior $a_{T}$ in the inset of Fig. 11(a) provides an activation energy of $7 \mathrm{kcal} \mathrm{mol}^{-1}$. This corresponds to the hindered rotation of the phenyl ring side chains about their bond with the backbone [72], also referred to as the $\gamma$-relaxation [73]. Above $T_{g}$, the Arrhenius representation of $a_{T}$ in the inset of Fig. 11(b) provides an apparent activation energy of $88 \mathrm{kcal} \mathrm{mol}^{-1}$, which coincides with the $90 \mathrm{kcal} \mathrm{mol}^{-1}$ energy barrier for the $\alpha$-relaxation of the PS backbone [74]. The WLF behavior expected above $T_{g}$ predicts a temperature dependent activation energy for the $\alpha$-relaxation, Eq. 9. In the WLF formalism, the constants $C_{1}$ and $C_{2}$ depend on the chosen reference temperature, $T_{R}$, used for the superposition [9]. For $T_{R}=388 \mathrm{~K}$, the experimentally determined values are $c_{1}=16$ and $c_{2}=114$. The corresponding activation energy at $T_{g}$, by Eq. 10 , is $89 \mathrm{kcal} \mathrm{mol}^{-1}$, which is close to the $88 \mathrm{kcal} \mathrm{mol}^{-1}$ value deduced from the Arrhenius representation in the inset of Fig. 11(b). The activation energies represent the potential barrier that is continuously overcome during steady sliding of the FFM tip. Thus, the molecular origin for polymeric friction lies in the rheological relaxation processes that are available in a given temperature range.

The qualitative difference in $\left.F_{F}(v)\right|_{T}$ curves below and above $T_{g}$ is insignificant; a bell-shaped friction-velocity behavior can be expected in both regimes. Under ideal conditions, it is only a question of the accessible velocity range. Ludema and Tabor [66] explained the $F_{F}(v)$ peak with respect to the variation of the contact area and the shear strength with scan velocity. Thus, friction expressed as $F_{F}\left(a_{T} v\right)=\sigma\left(a_{T} v\right) A[E(v)]$ will exhibit a bell-shape curve similar to Fig. 11(b), where $\sigma$ represents the shear stress, $A$ the real contact area, and $E(v)$ the viscoelastic modulus.
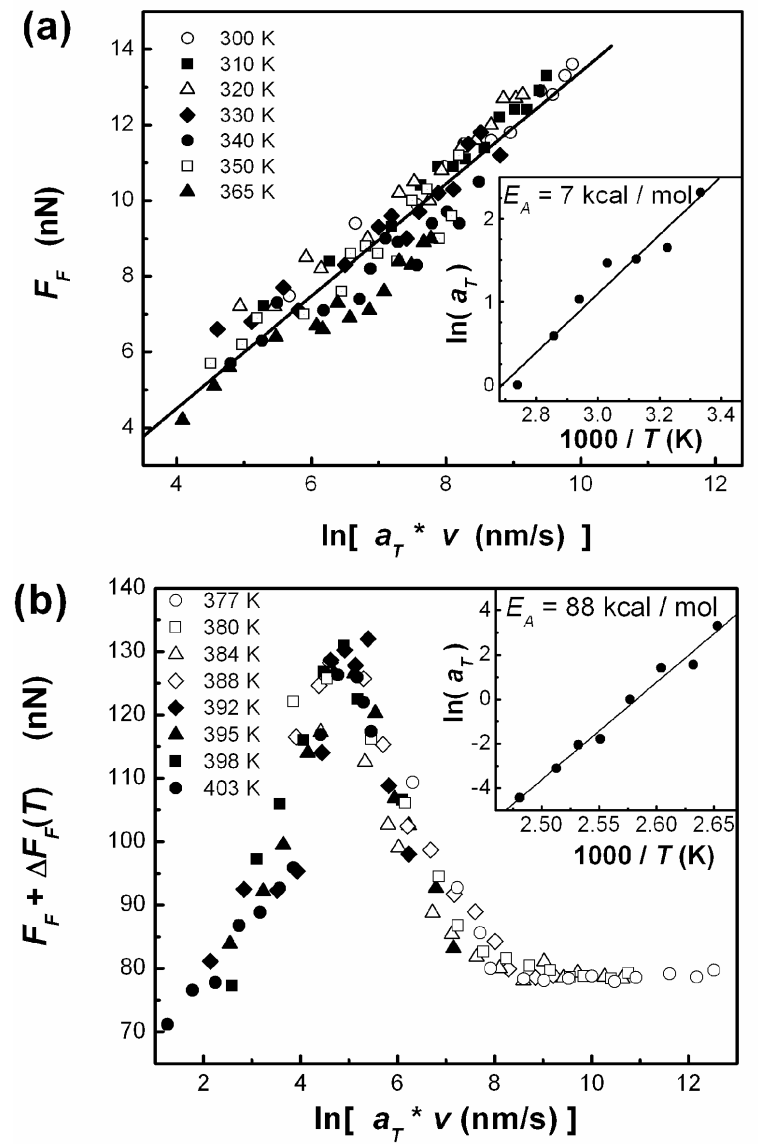

Figure 11. Friction master curves for atactic polystyrene: (a) Superposition below $T_{g}$ with (inset) corresponding $a_{T}$ shift factor (b) Superposition above $T_{g}$, with (inset) corresponding $a_{T}$ shift factor.

On the molecular scale, the qualitative rate behavior of both the shear strength and modulus, and thus the shape of the $F_{F}(v)$ curve, originates from the interplay of two dominating time scales: (i) the extrinsic drive time, $\tau_{e}$, dictated by the sliding velocity, $v$, and (ii) the intrinsic material response time, $\tau_{m}$, which in this case is the $\alpha$-relaxation time. In the vicinity of the $F_{F}(v)$ peak, the two competing processes occur on comparable time scales. The friction force increases or decreases with increased sliding velocity, depending on whether the extrinsic time leads or trails the material response time, respectively. Generally the interplay between intrinsic and extrinsic times is discussed with their ratio, the Deborah number. Since the material response above $T_{g}$ has been identified as the $\alpha$-relaxation, 
the particular $F_{F}(v)$ peak pictured in Fig. 11(b) is distinguished as the $\alpha$-peak.
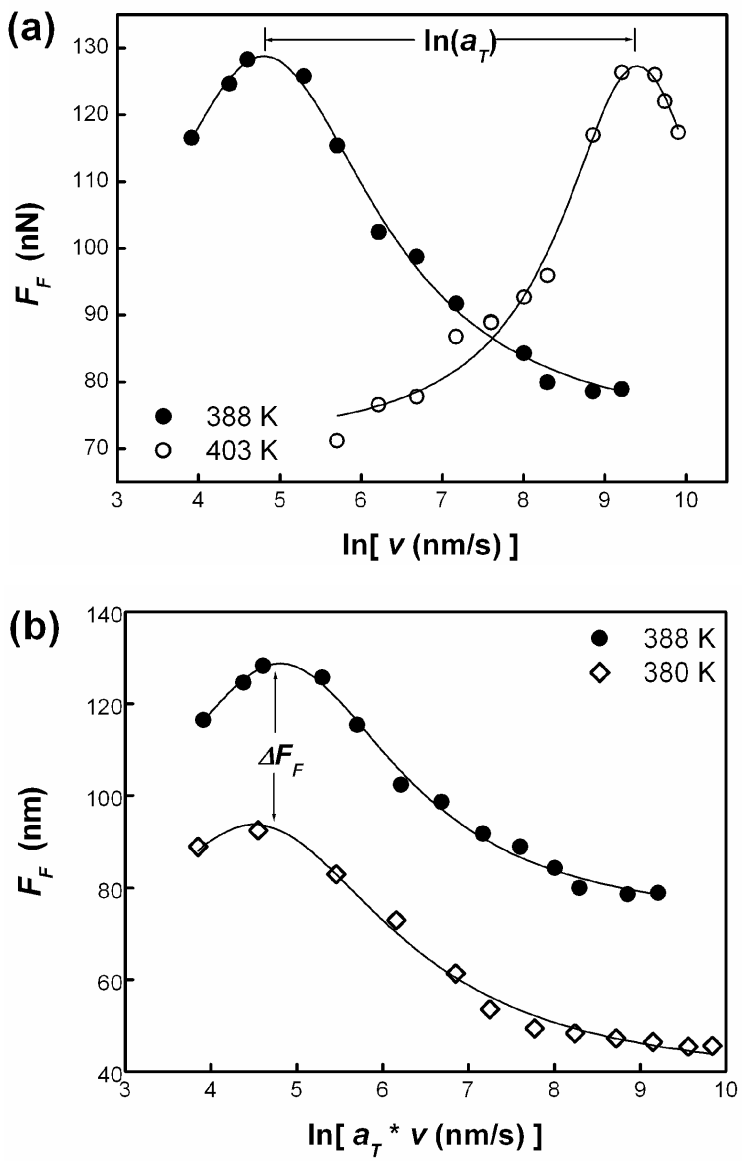

Figure 12. Representative shifts for superposition of friction data: (a) The horizontal shift applied all $F_{F}(v)$ isotherms defines the temperature dependent $a_{T}$ function. (b) The vertical shift, $\Delta F_{F}(T)$, is only necessary for $F_{F}(v)$ isotherms between $T_{g}=373 \mathrm{~K}$ and $T_{c}=388 \mathrm{~K}$.

No bell-shaped friction-velocity isotherms are observed below $T_{g}$, because reaching the time-scale for phenyl rotations would demand high sliding speeds of $\mathrm{mm} \mathrm{s}^{-1}$ to $\mathrm{cm} \mathrm{s}^{-1}$, unachievable with conventional SPM.

\subsection{Structural Heterogeneity}

While the energetics associated with the dissipation process are deduced from the horizontal $a_{T}$ shift, dynamical and structural information are inferred from the friction peak intensity, similar to spectroscopic techniques. The friction analysis below $T_{g}$ only involved classical horizontal shifting, which defines $a_{T}$ in Fig. 12(a). Additional vertical shifts, $\Delta F_{F}$, are necessary in the transition region between $T_{g}$ and $T_{g}+15 \mathrm{~K}$, Fig. 12(b). The application of vertical data shifts must be considered thoughtfully. Inspection of the $\alpha$-peak intensity, $F_{F, \max }(T)$, in Fig. 13 reveals the maturation of the $\alpha$-relaxation from $T_{g}$ to $T_{g}+15 \mathrm{~K}$. The strong $4.8 \mathrm{nN} \mathrm{K}^{-1}$ temperature dependence of $F_{F \text {, max }}$ in the transition regime is caused by the heterogeneity of two structural phases. Conceptually, small domains of the melt phase begin to appear at $T_{g}$, yielding a relatively weak $\alpha$-peak intensity. As the temperature increases, remnant glassy domains, or threads, are consumed by the melt; thus the $\alpha$-peak intensity increases. Once the melt phase is fully developed at $T>T_{g}+15 \mathrm{~K}$, a temperature independent $\alpha$-peak intensity is observed. Hence, the vertical shift used in Fig. 11(b), $\Delta F_{F}(T)$, represents the departure from equilibrium of a sturcturally heterogeneous melt.

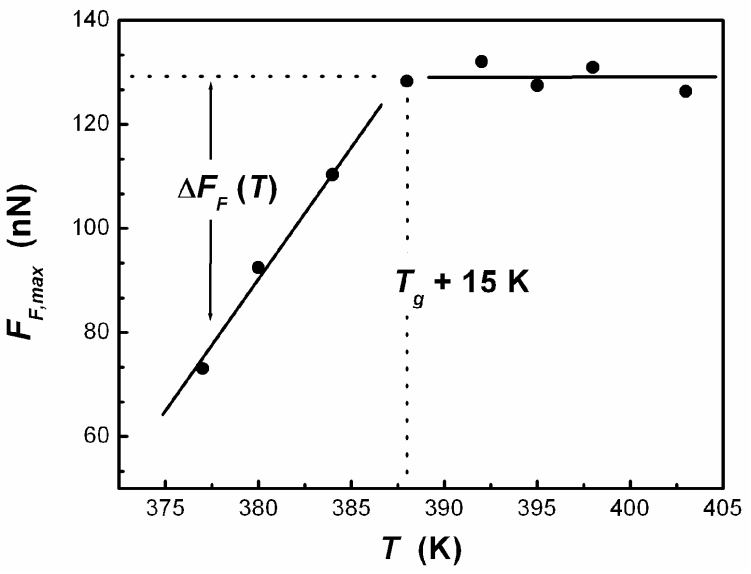

Figure 13. Friction peak intensity, $F_{F, \max }(T)$, for the $\alpha$-relaxation in atactic polystyrene. $\Delta F_{F}(T)=F_{F, \max }\left(T>T_{g}+15\right)-F_{F, \max }(T)$.

The above behavior concurs with the present understanding of vitrification between the crossover temperature $T_{c}$ and $T_{g}$, where $T_{c}>T_{g}$ [2]. Above $T_{c}$, glass forming polymers exhibit a single relaxation process. As the temperature is reduced below $T_{c}$, bifurcation of the relaxation process leads to both slow ( $\alpha$-backbone) and fast (side chain) relaxation processes $[75,76]$. As $T_{g}$ is approached, the slow process becomes locked; while the fast process continues below $T_{g}$ [75]. The onset of this bifurcation is evident in $F_{F, \max }(T)$ at $388 \mathrm{~K}$ in Fig. 13. Here, the $\alpha$-peak intensity, $F_{F, \max }(T)$, reveals an impedance of the $\alpha$-relaxation on cooling below $388 \mathrm{~K}$. Only the $\alpha$-peak is observed with conventional SPM (Fig. 11(b)); as noted above, the time-scale for relaxation of the phenyl side chains is not accessible. However, the transition from side chain to backbone relaxation is apparent in the friction coefficient in Fig. 10. The friction coefficient reveals three regimes: (i) a single glassy phase below $T_{g}$, where dissipation occurs only through phenyl rotations; (ii) a structurally heterogeneous system within $15 \mathrm{~K}$ above $T_{g}$, where dissipation occurs through a combination of spatially distributed fast (phenyl) and slow (backbone) relaxations; and (iii) a homogeneous melt above $388 \mathrm{~K}$, with dissipation solely through the higher energy $\alpha$-relaxation. 


\subsection{Cooperative Molecular Motion}

The existence of structural and dynamic heterogeneity around $T_{g}$ is consistent with conclusions drawn from isothermal multidimensional nuclear magnetic resonance (NMR), dielectric spectroscopy, photobleaching, dynamic light scattering, and quasi-elastic neutron scattering studies [2]. Since Adam and Gibbs [77], structural relaxation near the glass transition is visualized in terms of a correlated motion of polymer segments or domains, giving rise to dynamic heterogeneities [2, 75, 78-80]. While the time scale of dynamic heterogeneities can be directly inferred from scattering experiments, the size of the cooperatively rearranging regions (typically $1-3 \mathrm{~nm}[2,78,81,82]$ ) generally has not been directly obtainable and involves model assumptions. In particular, a temperature-resolved description of the cooperation length is expected to provide vital microscopic information towards the ongoing mysteries of the glass transition [78]. An unambiguous evaluation of this small length scale warrants a direct, spatially nanoscopic investigation of the glass forming dynamics, avoiding the averaging effects associated with conventional ensemble measurements [83].

The extent to which neighboring chain segments and adjacent molecules participate collectively in the dissipation, or relaxation, process is deduced from the intrinsic characteristics of the friction peak in Fig. 11(b). In early evaluations of the dissipation length in elastomers, Grosch [65] and Ludema and Tabor [66] combined the velocity at the friction peak with the frequency for the maximum viscoelastic loss, and deduced a length scale on the order of 5-10 nm. Similarly, the friction-velocity results in Fig. 11(b) are related to dielectric spectroscopy data [84] for PS of comparable molecular weight $\left(M_{w}=90.0 \mathrm{k}\right.$, $M_{w} / M_{n}=1.06$ ). It is founded that interpretations of dielectric relaxations are related by Eq. 43 to length scales involved in mechanical relaxation experiments [85]. With the specific knowledge of the $\alpha$-relaxation times $\tau_{\alpha}(T)$ from the dielectric data and the critical velocities $v_{o}(T)$ corresponding to the friction peaks, the length scale over which energy is dissipated during an $\alpha$-relaxation event may be expressed as:

$$
\xi_{\alpha}(T)=v_{o}(T) \cdot \tau_{\alpha}(T)
$$

The dissipation lengths $\xi_{\alpha}(T)$ for polystyrene are presented in Fig. 14. In the fully developed melt $(T>400 \mathrm{~K})$, a lower limit of $\sim 0.4 \mathrm{~nm}$ for $\xi_{d}(T)$, corresponds to the relaxation of individual monomer segments. On cooling from $403 \mathrm{~K}$ to $384 \mathrm{~K}$, the dissipation length increases steadily from 0.4 to $2.1 \mathrm{~nm}$, following a power law relation of the form $\left(T-T_{g}\right)^{-\phi}$, where $\phi$ is $1.86 \pm 0.09$, Fig. 14 . The thermal behavior of $\xi_{d}(T)$ confirms recent predictions based on molecular dynamics (MD) simulations $(\phi=1.87 \pm 0.15)$ for the spatial correlation of segmental displacements above the critical temperature of the mode coupling theory [86]. In essence, the dissipation length may be considered as the size of cooperatively rearranging regions (CRRs), or regions in space where the motion of individual chain segments is contingent upon the orchestrated motion of an ensemble of surrounding segments.

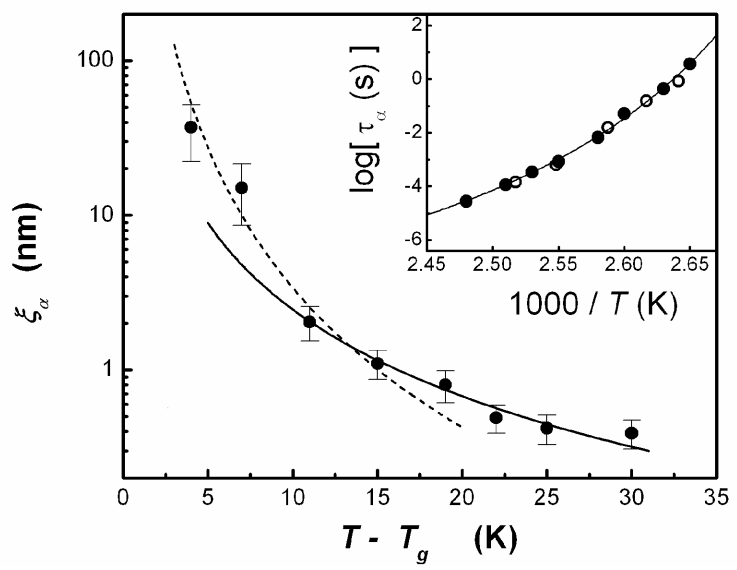

Figure 14. Polystyrene dissipation lengths for the $\alpha$-relaxation follow the power law relation $\xi_{\alpha} \sim\left(T-T_{g}\right)^{-\phi}$ with $T_{g}=373 \mathrm{~K}$. The exponent $\phi=1.86 \pm 0.09$ for $T \geq 384 \mathrm{~K}$ (solid line) and $\phi=3.0 \pm 0.2$ for $T \leq 388 \mathrm{~K}$ (dashed line). Inset: Corresponding polystyrene $\alpha$-relaxation times, $\tau_{\alpha}$, determined from the FFM friction peaks and Eq. 43 (closed circles) compared to dielectric spectroscopy measurements of [84] (open circles).

On closer approach to $T_{g}(T<388 \mathrm{~K})$, we find a strong deviation from the above power law behavior. Dissipation occurs in CRRs with sizes increasing up to $37 \mathrm{~nm}$. Recent MD simulations incorporating angular and torsional potentials, suggest that intramolecular interactions effectively slow down the cooperative dynamics near $T_{g}$ [87]. The MD simulation predicts an amplified power-law behavior with an exponent $\phi$ of 2.9 (ref [87]). A power law fit to $\xi_{\alpha}(T)$ below $388 \mathrm{~K}$ (Fig. 14) reveals an exponent $\phi$ of $3.0 \pm 0.2$. Based on this MD simulation, $388 \mathrm{~K}$ is interpreted as a critical temperature below which intramolecular interactions alter the dynamics of the CRRs, and thus, the relaxation behavior and the nature of the dissipation process [88]. In context of the observed power law behavior, $\xi_{\alpha} \sim\left(T-T_{g}\right)^{-\phi}$, the dissipation length $\xi_{\alpha}$ represents the degree of molecular coordination. The exponent $\phi$ captures the growth dynamics of the CRRs when approaching $T_{g}$, which itself, acts as a thermal asymptote to a diverging $\xi_{d}(T)$. This interpretation is consistent with the kinetic model of the glass transition in Section 2.

The large size of the CRRs deserves particular attention. The size of the CRRs in PS near the glass transition grows from single molecular segments to domains of tens of nanometers in diameter. Compared to structures in modern device technologies, e.g. ultrathin films and nanocomposites involving sub-100 $\mathrm{nm}$ dimensions, one can expect a competition between material and device length 
scales. Consequently, material properties in dimensionally constrained systems are likely to be modified from their original bulk values [89], which is the topic discussed in the following section. The impact of dimensional constraints on the CRR growth dynamics, $\phi$, will dictate the thermal range over which the glass transition occurs. Constraints leading to enhanced coordination (high $\phi$ - more restricted mobility) would increase the local $T_{g}$; while finite size effects that prevent coordination (low $\phi$ - less restricted mobility) would reduce the local $T_{g}$. Given the technological importance of $T_{g}$, the continued evolution of thin film applications stands to benefit from accurate characterization of $\xi_{\alpha}(T)$ and $\phi$ in confined geometries. Not only frictional dissipation, but all transport processes are hinged upon the same intra- and inter-molecular degrees of freedom available to particular motions, e.g. charge carrier transport in organic thin film transistors, light emitting diodes, and molecular electronic devices.

\section{Constraints and Structural Modifications near Interfaces}

The discussion thus far has focused on SPM techniques and bulk-material behaviors. In polymer thin films, when film thicknesses approach the nanometer scale, structural, material, and transport properties become increasingly dominated by interfacial and dimensional constraints. Rheologically modified boundary layers are often formed at interfaces, within which, anisotropic constraints lead to bulk-deviating behaviors. This section is devoted to exploring rheological boundary layers at polymer interfaces. A variety of material responses are illustrated with several FFM and SM-FM studies, and a visualization of the molecular configuration at interfacial boundaries is gradually developed with each successive example.

\subsection{Interfacial Plasticization}

The conceptually intuitive process of heterogeneous diffusion serves as worthy starting point for a discussion about rheological modifications at interfaces. This is illustrated for a multiphase, binary thin film system, in which, low molecular weight components (LMCs) leach from an underlying film into the surface film, forming an interdiffusion zone at the interface. FFM studies were conducted on poly(methyl methacrylate) (PMMA) films supported on either crosslinked epoxy or silicon substrates.

For PMMA films on epoxy substrates, the friction coefficient, $\mu$, in Fig. 15 decreases with increasing film thickness. For thicker films, $\mu$ approaches the friction value of PMMA on silicon. The friction coefficient may be considered constant, if one assumes that (a) the shear modulus is constant, and (b) the adhesion force is only a function of the contact area, i.e. a constant physical and chemical bonding strength between the FFM tip and sample.
Holding to these assumptions and considering that the FFM probe (silicon) is much stiffer than PMMA, it follows that changes in $\mu$ reflect changes in the PMMA modulus.

The friction coefficient for the $35 \mathrm{~nm}$ films is significantly higher with epoxy substrates than for silicon substrates. However, the friction coefficient on thicker epoxy supported films reaches the low value found on the thin, $35 \mathrm{~nm}$ silicon supported film, appearing substrate independent. The friction gradient suggests leaching of LMCs from the epoxy into the PMMA, illustrated in Fig. 15, essentially softens, or plasticizes, the film and reduces the PMMA modulus.

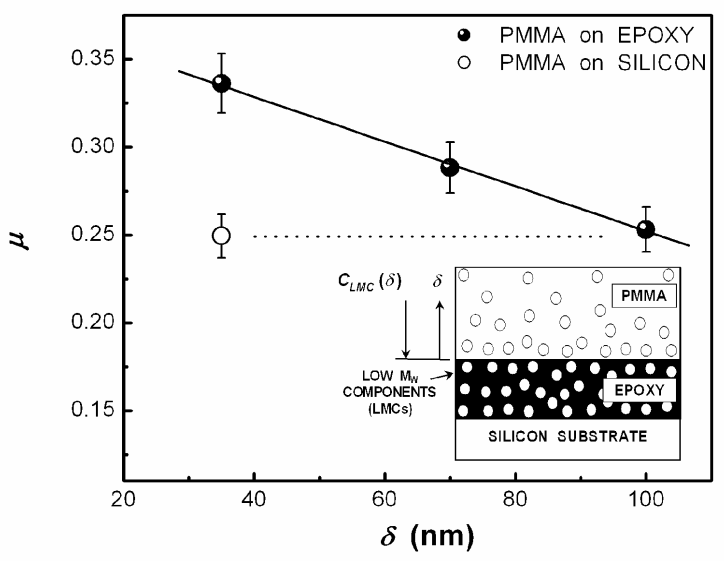

Figure 15: FFM friction coefficient measurements on PMMA reveal interfacial plasticization for films supported on epoxy substrates, as low molecular weight components leach from the epoxy into the PMMA.

The extent of the softening, and modulus depression, is proportional to the concentration of LMCs $\left(C_{L M C}\right)$ at the surface, which in turn, is a function of film thickness, $\delta$. At a thickness of $100 \mathrm{~nm}$, the friction coefficient matches that of the $35 \mathrm{~nm}$ Silicon supported PMMA, indicating no detectable plasticization, or LMCs, at the surface. This interdiffusion across interfaces highlights the importance of substrate chemistry for thin film applications. In this case, a $100 \mathrm{~nm}$ thick boundary layer is rheologically modified due to the plasticization effects of interdiffused low molecular weight components.

\subsection{Dewetting Kinetics}

In the prior discussion, chemical transport processes were responsible for rheological modifications. The impact associated with physical and dimensional constraints is perhaps, less intuitive. Never the less, various groups have reported bulk-deviating structural and dynamic properties for polymers at interfaces [13, 90-93]. For example, reduced molecular mobility in ultra-thin PS films was reported based on forward recoil spectroscopy measurements [13]. 
The contribution of the substrate to rheological modifications becomes apparent in dewetting studies with binary films of PS on polyethylene-co-propylene (PEP), which are supported on silicon substrates (high interaction surface) [90]. The dewetting kinetics in Fig. 16 were determined from a time-series of SPM topography images, and reveal a critical PEP film thickness, $\delta_{C R I T}$, below which, the dewetting velocity $\left(V_{d}\right)$ decreases with decreasing film thickness, and above which, $V_{d}$ remains constant. Independent FFM measurements on silicon supported PEP films also indicate a critical film thickness, $\delta_{C R I T}$, below which, the friction decreases with decreasing film thickness, and above which remains constant. In both studies, the critical PEP film thickness in Fig. 16 corresponds to approximately $100 \mathrm{~nm}$.

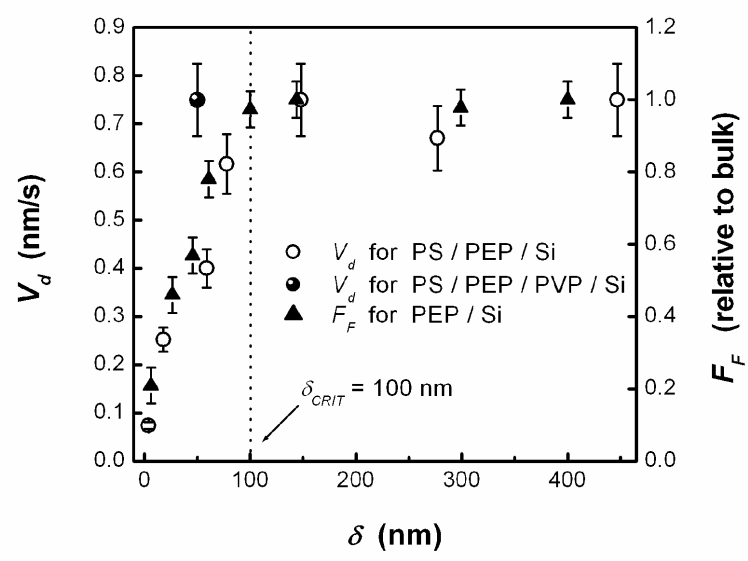

Figure 16: Dewetting velocity $\left(v_{d}\right)$ and friction $\left(F_{F}\right)$ measurements on PS/PEP systems reveal a $100 \mathrm{~nm}$ interfacial boundary layer. Data from reference [90].

The dewetting kinetics and friction forces both suggest the presence of a rheologically modified PEP boundary adjacent to the silicon interface. For $\delta_{P E P}<\delta_{C R I T}$, the decreasing friction represents an increase in the PEP modulus. This translates to an increasing glasslike behavior, or loss of mobility, as the silicon interface is approached through the PEP phase. It is this loss of PEP mobility that is responsible for decreasing the dewetting velocity.

To identify the source of this rheological gradient, the PEP-Silicon interactions were effectively masked by first spin casting a low interaction foundation layer of poly(vinyl pyridine) (PVP) on the silicon. The dewetting velocity of the PS/PEP/PVP film is reported as the open box in Fig. 16 and remained constant, even at PEP film thicknesses below $\delta_{C R I T}$. This anomalous finding unveils the high interfacial interactions between PEP and silicon as responsible for the apparent PEP vitrification inside the interfacial boundary.

\subsection{Disentanglement Barriers}

The current picture of the rheological boundary attributes its formation to interfacial constraints on the molecular mobility. In the past, interfacial effects were considered to be confined to the pinning regime, typically on the order of a few nanometers. However, FFM disentanglement studies on PEP films [94] and NMR tracer diffusion measurements in PS [92] have revealed that the interfacial boundary may extend up to 10 radii of gyration $\left(R_{G}\right)$ beyond the interface. Simple surface pinning alone has been ruled out since, at this distance, the probability of a polymer molecule making direct surface contact is nearly zero.

FFM friction measurements on Silicon supported PEP films $\left(R_{G}=24 \mathrm{~nm}\right)$ offer insight to the source of these farfield molecular constraints. A transition in the friction coefficient at a critical load $(P)$ is seen in Fig. 17. The higher friction coefficient below $P$ portrays a dissipative behavior consistent with viscous plowing through an entangled PEP melt.

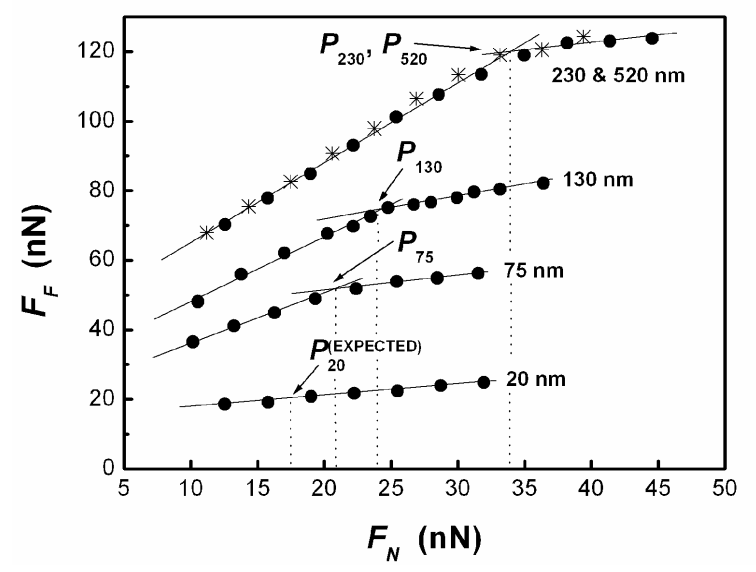

Figure 17: FFM measurements on PEP films reveal a critical load $(P)$ marking a transition from viscous shearing to chain sliding. Data are from reference [94].

At loads exceeding $P$, the reduced friction coefficient represents a chain slipping phenomenon similar to a shear banding behavior. Thus, the critical load may be conceptualized as an effective activation barrier for disentanglement [94]. The boundary layer thickness and information about to the conformational structure within the boundary are elucidated from the film thickness dependence of $P$ :

(i) The absence of the disentanglement transition $(P)$ in the $20 \mathrm{~nm}$ films and the ubiquitous low friction, chain slipping suggest that the PEP molecules are highly disentangled within a sublayer immediately adjacent to the substrate.

(ii) In the $75-230 \mathrm{~nm}$ films, the disentanglement transition $(P)$ increases linearly with film thickness until the bulk $P$ is reached. The sub-bulk $P$ values indicate an intermediate regime of partial disentanglement, the extent of which diminishes with increasing film thickness until the bulk entanglement density is recovered. This far-field 
disentanglement ( $\sim 10 R_{G}$ from the substrate) is attributed to the strain imposed during spin casting. The preservation of the disentangled structure in the melt reflects an anisotropic diffusion process where partially disentangled chain ends diffuse into the more porous structure [92] of sublayer [94].

(iii) Finally, for films thicker than $230 \mathrm{~nm}$, the polymer behaves like the bulk elastomer and loses any memory of the underlying silicon.

The picture of rheological boundary layers now reflects a two phase system comprised of a sublayer and an intermediate regime. The mobility constraints are ascribed to the strain imposed during spin casting, paired with interfacial interactions in the sublayer and anisotropic diffusion in the intermediate regime.

\subsection{Interfacial Glass Transition Profiles}

It has been recognized that several factors are intricately responsible for the departure of $T_{g}$ in ultrathin films, from the bulk value [27, 44, 45, 95-99]; e.g. the proximity of a free surface, substrate interactions, and process-induced anisotropy. Here, we address the effects of spin casting on the interfacial $T_{g}$ profile of amorphous polymer films, along with the use of chemical crosslinking as a mobility control.

SM-FM $T_{g}$ measurements on PS films $\left(M_{w}=12 \mathrm{k}\right)$ are presented in Fig. 18. For film thicknesses, $\delta>200 \mathrm{~nm}$, the $T_{g}$ values correspond to the bulk $T_{g}$ of $95^{\circ} \mathrm{C}$ [24]. A two phase boundary layer is encountered within $\sim 200 \mathrm{~nm}$ of the substrate: (a) $T_{g}$ values are depressed relative to the bulk in a sublayer with a thickness on the order of $R_{G}$, i.e. one order of magnitude beyond the persistence length [100]; and (b) $T_{g}$ values exceed the those of the bulk in the intermediate regime.

This non-monotonic $T_{g}(\delta)$ relationship is interpreted considering two competing processes that affect the relaxation dynamics: (a) shear induced structuring and (b) interdiffusion [92, 94, 101]. Shear structuring creates an interfacial region where the spin casting shear stresses induce polymer stretching and or disentanglement (structural deformation). The second process involves the interdiffusion between the entropically cooled interfacial region and the unperturbed bulk phase.

With a strong precedence for shear structuring in PS solutions $[102,103]$ and considering the shear stress profiles during spin casting [104], it is reasonable to propose that the effects of spin casting extend from the substrate to the boundary with the bulk phase. In this scenario, the extent of structural deformation is related to the shear stress profile during casting. Alternatively, the shear structuring may extend only through the sublayer, and interdiffusion alone may be responsible for the conformational restructuring in the intermediate regime. For this case, the molecular mobility is limited by the propagation of holes, or packets of free volume, which facilitate conformational rearrangements [105].

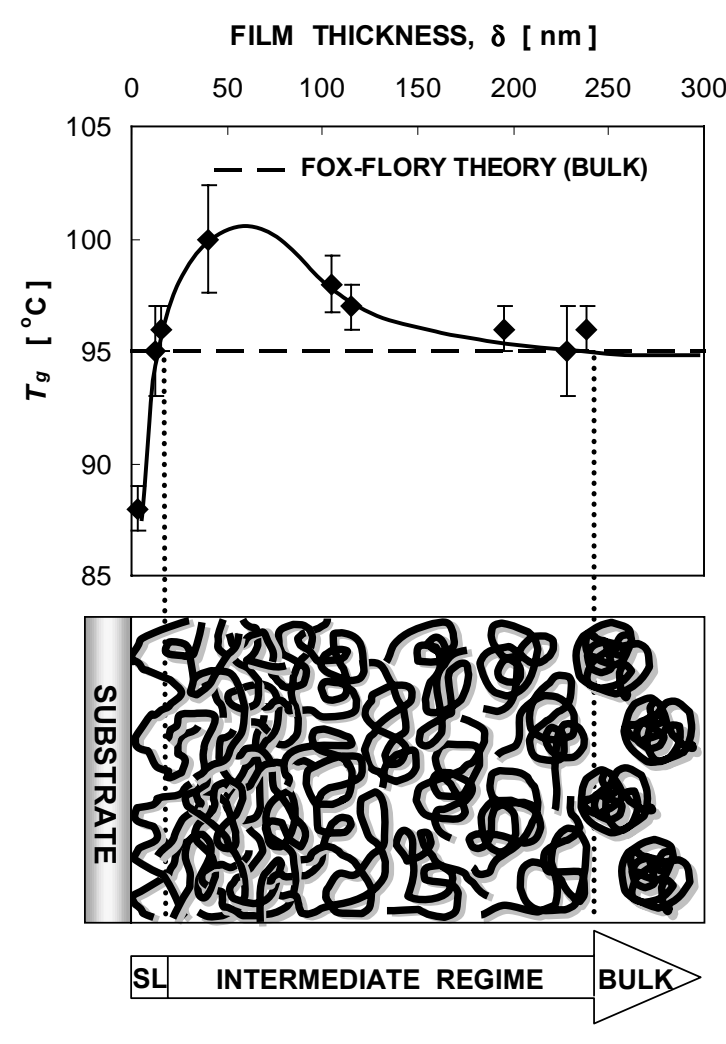

Figure 18. (top) Film thickness, $\delta$, dependence of $T_{g}$ for PS films $\left(M_{w}=12 \mathrm{k}\right)$ compared to the bulk $T_{g}$ from Fox-Flory theory. (bottom) Rheological boundary model for the observed $T_{g}(\delta)$ relation (SL=sublayer). Data from reference [89].

Spin casting films of increased molecular weight $\left(M_{w}\right)$ had the effect of shifting the $T_{g}(\delta)$ profiles further from the substrate, by $\sim 10 \mathrm{~nm} \mathrm{kDa}^{-1}$ [89]. The bulk $T_{g}$ is recovered at $\sim 250 \mathrm{~nm}$ for all films in the $M_{w}$ range of $(12-21 \mathrm{k})$. The influence of $M_{w}$ on the internal structure of the boundary layer appears more pronounced on the sublayer thickness than on the far-field boundary of the intermediate regime. This suggests that the overall boundary thickness depends more strongly on the spin casting shear stresses than on molecular dimensions.

When the molecular weight is increased by crosslinking pre-cast PS-BCB films, the $T_{g}(\delta)$ profiles exhibit a similar qualitative behavior before and after crosslinking [89], indicating a preservation of the rheological anisotropy after crosslinking at $250^{\circ} \mathrm{C}, \sim 150^{\circ} \mathrm{C}$ above $T_{g}$. The crosslinking yields an overall $T_{g}$ increase of $7 \pm 3{ }^{\circ} \mathrm{C}$; however, in contrast to the $M_{W}$ dependence discussed above, no spatial shift is found in the $T_{g}(\delta)$ profiles. Since crosslinking occurs after spin casting, the shear stresses that create the shifted $T_{g}(\delta)$ profiles are not present. Hence, the $T_{g}(\delta)$ profiles are impacted differently for each condition of increased $M_{w}$ because of the sequence of treatments.

The two phase model for rheological boundary layers has evolved to include interfacial interactions that lead to the formation of a less dense sublayer adjacent to the 
interface. The thickness of the sublayer is characterized, in part, by the molecular dimensions and the interaction potential at the interface. The coupled effects of shearinduced structuring during spin casting and anisotropic relaxation and transport constraints during annealing are responsible for the creation of an intermediate regime between the sublayer and bulk phase. The overall rheological boundary may extend up to two orders of magnitude beyond the polymer's persistence length, and the molecular restructuring within the boundary is thermally stable well above $T_{g}$. Finally, the impact of mobility constraints (e.g. crosslinking) on the structure within boundary layers depends on the sequence of the film preparation process, i.e. constrains incorporated before and after casting exhibit different rheological outcomes. Given the technological relevance of spin casting, the effect of structural modifications in nanoscopic systems is seen with wide interest, across many disciplines. In what follows, we explore how rheological constraints are encountered in some of the mechanical operations involved with nano-electromechanical systems (NEMS).

\section{Mechanical Operations in Nanoscopic Polymer Systems}

The length scales for cooperative molecular motion in Section 4 and for interfacial boundaries in Section 5, ranged from tens to roughly one hundred nanometers. Compared to structures in modern device technologies, e.g. ultrathin films and nanocomposites involving sub-100 nm dimensions, one can expect a competition between material and device length scales. We have demonstrated above, how material properties in confined geometries may be modified from their original bulk values. At this point, one must anticipate the extent to which such rheological modifications contribute to current technological challenges.

Mechanical operations have traditionally been one of the most important pathways for technological evolution. Normally, the goal lays in maintaining control of some particular motion. Currently, one concern deals with the implication of finite size effects in the contact mechanics associated with nano-electromechanical (NEMS) applications. Particularly relevent is the process of scanning probe, thermomechanical data storage [3, 106]

Thermomechanical data storage (TDS) relies on writing, reading, and erasing nanometer sized data bits in thin polymer films, offering densities up to $\mathrm{Tb} \mathrm{in}^{-2}[3,106]$ In essence, the TDS writing operation is a high speed $(\mathrm{MHz})$, elastic-viscoplastic polymer indentation process, Fig. 19. The polymer storage media must be designed to achieve the narrow range of physiochemical properties necessary for: high data density, fast data rates, high durability, long shelf life, and low power consumption. The ideal polymer should be easily deformable for bit writing; however, the written bits must be stable against dewetting, thermal degradation, and wear.
Each indented bit represents a metastable state of the deformed volume, and will either initiate spontaneous dewetting (film instability) or strive for recovery of the initial unstressed state (bit instability) [107]. The delicate balance between these instability nodes constitutes one optimization scenario in the design of polymeric storage media. Furthermore, media (and data!) wear must be minimized during scanning operations. In particular, topographical protrusions, in the form of piled-up rims around the indented bits, are regions susceptible to wear. The presence of rims also adversely affects the writing density. Rims interact non-linearly with adjacent bits, lowering the signal-to-noise ratio of bit detection and requiring a relaxation of the indentation pitch (data density). From the perspectives of media wear and data density, a suitable polymer storage media exhibits a weak propensity for rim formation during indentation.

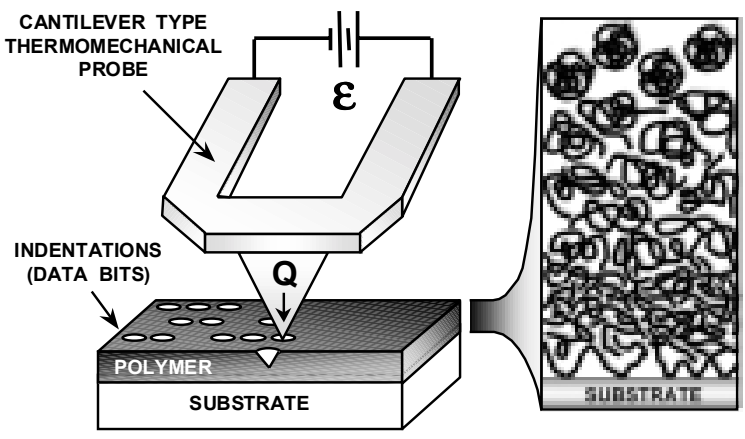

Figure 19. Scanning Probe, Thermomechanical Data Storage: Creating uniform bit indentations in sub$100 \mathrm{~nm}$ thick polymer films requires an understanding of the interfacial rheology.

In this section, we explore how the material modifications associated with dimensional constraints make themselves apparent during nano-contacting operations. We investigate strain shielding at the substrate interface, and its implications on rim formation, during high strain-rate indentations in thin polystyrene films. Further, the role of material anisotropy in the distribution of indentation loads will be elucidated with the interfacial $T_{g}$ profiles from Section 5. First, let us commence with a theoretical background for the contact mechanics associated with the isotropic bulk-material case.

\subsection{Indentation Contact Mechanics}

During normal indentation of an elastic-plastic material, when the yield point of the more ductile material is first exceeded, the onset of plastic (anelastic) deformation commences. Initially, the plastic region is small and completely contained by the surrounding elastic material. Hence, the plastic strains are of the same order of magnitude as the surrounding elastic strains. The plastically displaced material is fully accommodated by elastic expansion of the 
surrounding solid. This is referred to as confined deformation because the flowing or plastically deforming volume is fully constrained by the surrounding elastic medium. As the applied strain is increased, i.e. increased load or sharper indenter, a greater pressure beneath the tip is required to produce the necessary expansion. Eventually, sufficient pressure is achieved where the plastic region reaches the free surface, allowing the displaced material to escape via unconfined plastic flow along the sides of the indenter.

The onset of plastic yield, or confined deformation, is assessed by applying an appropriate yield criterion. The two most commonly applied criteria are the Tresca's maximum shear stress criterion, where yielding occurs when the maximum shear stress, or half the difference between the maximum and minimum principle stresses, reaches the yield stress in pure shear or half the yield stress in simple compression (or tension) [38]:

$$
\begin{gathered}
\max \left[\frac{1}{2}\left|\sigma_{1}-\sigma_{2}\right|, \frac{1}{2}\left|\sigma_{2}-\sigma_{3}\right|, \frac{1}{2}\left|\sigma_{3}-\sigma_{1}\right|\right] \\
=k=\frac{Y}{2}
\end{gathered}
$$

and the von Mises' shear strain-energy criterion, where yielding occurs once the deformation energy equals the deformation energy at yield in simple compression or pure shear [108]. Therefore, by the von Mises criterion, yielding occurs when the square root of the second invariant, $J_{2}$, of the stress deviator tensor, $S_{i j}$, reaches the yield stress in simple shear or $1 / \sqrt{ } 3$ of the yield stress in simple compression:

$$
\begin{gathered}
\sqrt{J_{2}}=\left[\frac{1}{2} S_{i j}\right]^{1 / 2}= \\
\left\{\frac{1}{6}\left[\left(\sigma_{1}-\sigma_{2}\right)^{2}+\left(\sigma_{2}-\sigma_{3}\right)^{2}+\left(\sigma_{3}-\sigma_{1}\right)^{2}\right]\right\}^{1 / 2} \\
=k=\frac{Y}{\sqrt{3}}
\end{gathered}
$$

where $\sigma_{1}, \sigma_{2}$, and $\sigma_{3}$ are the principle stresses in the state of complex stress, and $k$ and $Y$ represent the yield stresses in pure shear and simple compression (or tension). For detailed analysis of the state of complex stress and formulation of the yield criteria, the reader is referred to [109].

Experiments on isotropic metals support the von Mises criterion over Tresca's; however, the discrepancy between the two is relatively small considering the variability in $k$ or $Y$ and the inherent anisotropies in most materials [38]. Therefore, it is generally acceptable to apply Tresca's criterion for its mathematical simplicity.

Relating the stresses from the yield criteria to the mean contact pressure under the indenter,

$$
p_{m}=c Y
$$

where, for the onset of constrained plastic yield, $c$ has a value of about 0.5 for conical indenters, and may vary depending on the indenter geometry and the friction at the interface [38].

The onset of unconfined plastic flow, i.e. the point when the plastic yield zone reaches the free surface, is expected to occur when the contact pressure reaches the yield stress given by rigid-plastic theory. Based on a number of numerical analyses and indentation measurements with rigid spheres and cones in elastic-plastic half-spaces, Johnson (1985) [38] has determined a value of $c \sim 2.8$, in Eq. 46.

In the transitional regime, when the contact pressure lies between $0.5 \sim 3 Y$, plastic flow is contained by the surrounding elastic material. The resulting deformation is generally in the form of radial expansion with roughly hemispherical contours of equal strain [38]. Based on these observations, Johnson (1970) [110] has developed a simple cavity model of elastic-plastic indentation, Fig. 20.

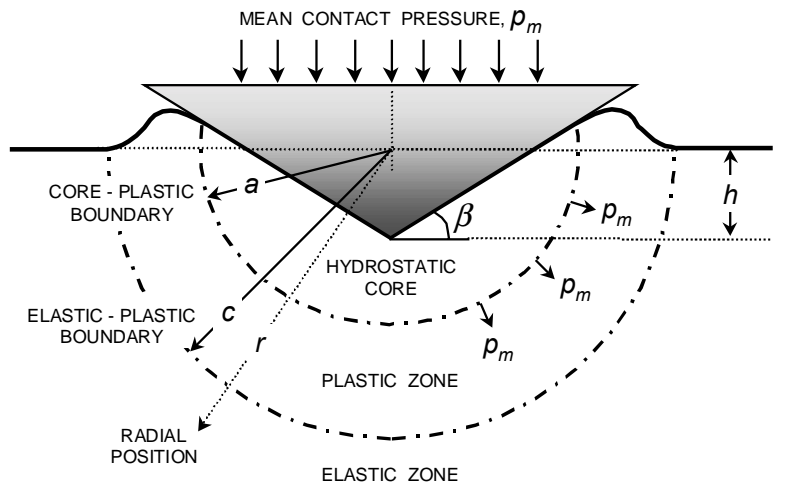

Figure 20: Cavity model of elastic-plastic indentation by a rigid cone. The mean contact pressure, $p_{m}$, directly beneath the contact is supported by a hydrostatic core with radius $a$. Beyond the core, unconstrained plastic deformation extends through the hemisphere where the pressure exceeds the yield stress by roughly three-fold [38]. The plastic front with radius $c$ is preceeded by elastic strain that accomodates pressures insufficient of producing yield.

The cavity model assumes that directly beneath the indenter contact surface, a hemispherical core with a radius equal to that of the projected contact area, $a$, has a hydrostatic stress component equal to the mean contact pressure, $p_{m}$. Immediately beyond the core lies the plastic zone, and at the core-plastic boundary, the radial stress component in the plastic zone equals the hydrostatic stress of the core. Within the plastic zone, stresses and displacements have radial symmetry, and the plastic strains gradually dimish with increasing radial distance, until they 
match the elastic strains at the elastic-plastic boundary, $c$ $(c>a)$.

Based on Hill [109], the stresses in the plastic zone, $a \leq r \leq c$, are characterized by:

$$
\frac{\sigma_{r}}{Y}=-2 \ln \left(\frac{c}{r}\right)-\frac{2}{3}, \quad \frac{\sigma_{\theta}}{Y}=-2 \ln \left(\frac{c}{r}\right)+\frac{1}{3}
$$

and in the elastic zone, where $r \geq c$

$$
\frac{\sigma_{r}}{Y}=-\frac{2}{3}\left(\frac{c}{r}\right)^{3}, \quad \frac{\sigma_{\theta}}{Y}=\frac{1}{3}\left(\frac{c}{r}\right)^{3}
$$

At the core-plastic boundary, the core pressure is given by:

$$
\frac{p_{m}}{Y}=\left.\left(-\frac{\sigma_{r}}{a}\right)\right|_{r=a}=2 \ln \left(\frac{c}{a}\right)+\frac{2}{3}
$$

Equation 49 implies that the elastic-plastic boundary coincides with the core-plastic boundary, $c=a$, at $p_{m}=$ ${ }_{2}^{2} Y$, and at reduced pressures, no plastic flow occurs. Therefore, the cavity model predicts that the onset of plastic yield occurs at $p_{m}={ }^{2} / 3 Y$ which is close to the value of $c \sim 0.5$ reported by Johnson (1970) [110]. The difference is attributed to $\beta$, and friction at the interface.

Radial displacement of matter at the core-plastic boundary, $r=a$, during an increment of penetration, $d h$, must accommodate the volume of material displaced by the indenter. Neglecting core compressibility, conservation of core volume requires:

$$
2 \pi \mathrm{a}^{2} \mathrm{~d} u(a)=\pi \mathrm{a}^{2} \mathrm{~d} h=\pi \mathrm{a}^{2} \tan (\beta) \mathrm{d} a
$$

The radial displacements within the plastic zone are given by [109]:

$$
\frac{d u(r)}{d c}=\frac{Y}{E}\left[3(1-v)\left(\frac{c}{r}\right)^{2}-2(1-2 v)\left(\frac{r}{c}\right)\right]
$$

Equations 50 and 51 are used to locate the elastic-plastic boundary, $c$, recognizing that for a conical indenter, geometrical similarity of the strain field with continued penetration requires $d c / d a=c / a=$ constant:

$$
\left(\frac{c}{a}\right)^{3}=\frac{1}{6(1-v)}\left[\frac{E \tan \beta}{Y}+4(1-2 v)\right]
$$

The core pressure is determined via substituting $c / a$ into Eq. 48 , and for an incompressible material, i.e. $v=0.5$ :

$$
\frac{p_{m}}{Y}=\frac{2}{3}\left[1+\ln \left(\frac{1}{3} \frac{E \tan \beta}{Y}\right)\right]
$$

The hydrostatic core pressure appears solely dependent on the parameter $(E / Y) \tan \beta$, which represents the ratio of the strain imposed by the indenter $(\tan \beta)$ to the strain capacity of the indented material $(Y / E)$. Generally, the indentation pressure under elastic, elastic-plastic, and fully plastic conditions is correlated as dimensionless contact pressure, $P_{m} / Y$, versus dimensionless strain, $(E / Y) \tan \beta$.

The above analysis was limited to elastic-perfectly plastic materials with a constant yield stress. Tabor has shown that the perfectly plastic analysis may be applied, with good approximation, to materials that strain hardening according to the power law relation:

$$
Y_{R}=\sigma_{o}\left(\frac{\varepsilon_{R}}{\varepsilon_{0}}\right)^{\frac{1}{n}}
$$

if $Y$ in Eq. 53 is replaced by a representative flow stress $Y_{R}$ measured at a representative strain $\varepsilon_{R}$, [111]. In Eq. 54, $n$ is the reciprocal of the work hardening index, and $\sigma_{0}$ is the work hardening coefficient. For a conical indenter, the representative strain is approximated by [38]:

$$
\varepsilon_{R} \approx 0.2 \tan \beta
$$

The scenario is somewhat more complicated for the case of viscoelastic plastic indentation. Many materials, notably polymers, exhibit viscoelastic behavior, which is characterized by a time and temperature dependent stressstrain relationship. The issue becomes one of determining the time dependence of the contact area and pressure distribution, which result from a prescribed loading. In cases where the corresponding solution for a purely elastic material is known, the simplest approach to this problem, based on Radok, consists of replacing the elastic constant with the corresponding integral operator from the viscoelastic stress-strain relations, i.e. the creep compliance or relaxation functions [38].

In the general isotropic case, $(E / Y) \tan \beta$ captures the essence of the mechanics, hence is referred to as the rheological factor $X$. With interfacial systems, where finite size effects may lead to material anisotropy, one has to be additionally concerned with the direction of the rheological gradients. We will show in Section 6.3.2, that the material response to indentation is distributed between two mechanical scenarios depending on the direction of the interfacial modulus and $T_{g}$ gradients. However, let us revisit the TDS recording process, and describe the outcome of the indentation process, i.e. a material's propensity to form piled-up rims (or to sink in), in terms of the rheological factor $X$.

\subsection{Rim Formation During Indentation}

During indentation of a rigid plastic solid, the displaced material appears in the piled-up rim around the periphery of 
the indentation site. With elastic-plastic materials, some, if not all, of the displaced material is accommodated by radial expansion of the elastic surroundings. Briscoe et al. have found for indentation and scratch hardnesses studies of PMMA, the measured yield stress is strongly dependent on both the indenter geometry (related to strain) and applied strain rate. During normal indentation with conical indentors of large excluded angles $\left(\beta=75^{\circ}\right)$, PMMA deforms by extrusion to the free surface with the creation of a piled-up rim. With blunter cones, i.e. lower $\beta$, deformation occurred elastoplasticly resulting in little or no pile-up [112]. In nanoscratch studies on PMMA, Adams et al. have demonstrated that the height of the pile-up will increase with $\tan \beta$ [113]. In a similar study on polycarbonate, Jardret et al. have observed distinctly different pile-up formations on nanoscratch samples of identical hardness. The difference in pileup height is attributed to the strain, with increased rim heights for larger strains, i.e larger $\tan \beta$ [114]. With a 2D finite element approach, Ramond-Angélélis has modeled piled-up rim formation in viscoelastic-perfectly plastic materials as a function of the rheological factor $X$. These results are presented in Fig. 21, and suggest for values of $X$ $<\sim 10$, the deformation during indentation is mainly elastic with little pile-up, and for $X>\sim 100$, the deformation is primarily plastic resulting in substantial pile-up [115].

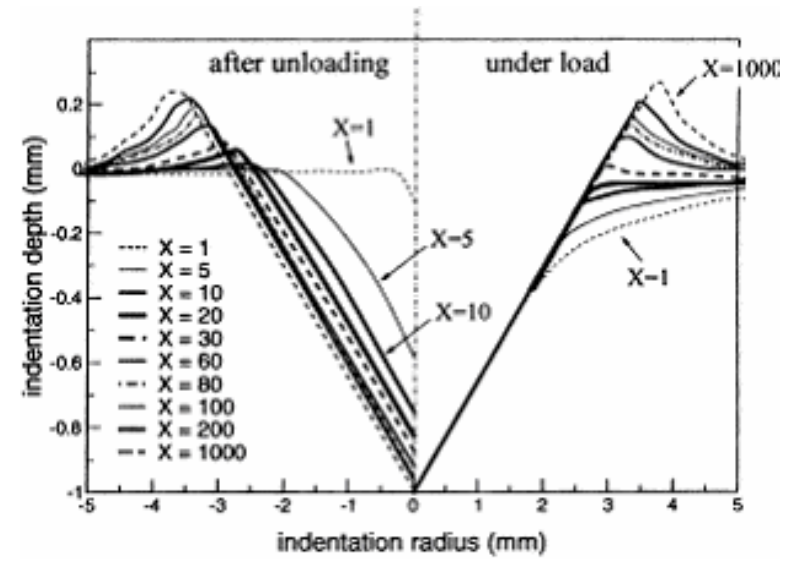

Figure 21: Cross-sectional view of elasto-plastic indentation under load and after unloading for a rheological factor, $X$, ranging from 1-1000. Results obtained by Ramond-Angélélis with a 2-D finite element model [115]

Pile-up formation in viscoelastic materials is also dependent on the strain rate. At high strain rates, PMMA displays a noticeable strain softening behavior that becomes more pronounced with increasingly higher strain rates and is absent at reduced strain rates $\left(<\sim 10^{-5} \mathrm{~s}^{-1}\right)$ [112]. Consequently, the yield stress decreases with increased strain rate, and is accompanied by the appearance of shear bands, which have been attributed to the onset of pile-up $[116,117]$. The effect of a high strain rate at large strains, i.e. high $\tan \beta$, may also induce adiabatic heating within the shear bands, which would promote large inhomogeneous strains, as well as, enhanced strain softening [112]. On the other hand, strain hardening tendencies also effect pile-up. A large capacity for strain hardening advances the plastic zone further into the material, thus decreasing pile-up adjacent to the indenter [118]. For an elastic-plastic material which strain hardens according the power law in Eq. 56, Matthews has proposed the indenter penetration depth, $h$, follows:[118]

$$
\frac{\zeta}{h}=\frac{1}{2}\left(\frac{2 n+1}{2 n}\right)^{2(n-1)}-1
$$

where $\zeta$ represents the vertical dimension with respect to the neutral surface of a piled-up rim, or sunken depression, at the periphery of the indentation, Fig. 22.

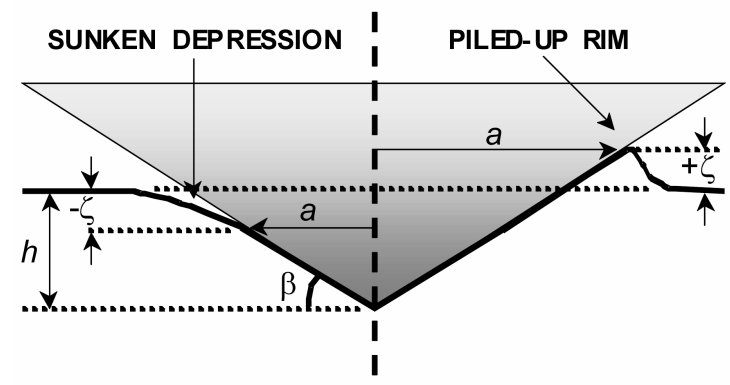

Figure 22. Peripheral deformation for opposing extremes of stress sensitivity. Sunken depressions versus piled-up rims for materials that exhibit strong versus weak strain hardening susceptibilities, respectively.

For a conical indenter:

$$
h+\zeta \cong a \tan \beta
$$

Combined with Eq. 56, the indentation depth becomes:

$$
h=2\left(\frac{2 n}{2 n+1}\right)^{2(n-1)} \operatorname{atan} \beta
$$

For $h /(a \tan \beta)>1$ (i.e. $n<3.8)$, a sunken depression is formed, and when $n$ exceeds $3.8, h /(a \tan \beta)<1$ and a piled-up rim is formed.

In viscoelastic materials, such as polymers, strain hardening should not be ignored. The pile-up formed during the nano-scratch studies on PMMA revealed a maximum hardness value at the rim apex, which decayed asymptotically to the hardness of the unperturbed film with increasing distance from the indentation site [113]. Both the rim height and the extent of strain hardening increased with the applied strain, i.e. $\tan \beta$ [113]. In addition, Adams et al. observed that the hardened pile-up of an existing scratch reduces the depth of a subsequent parallel scratch within the strain hardened area. This is important in the context of 
thermomechanical data storage where ultra high storage densities are sought by minimizing the pitch of indented data-bits. A tight indentation pitch with overlapping strain hardened zones would likely result in non-uniform indentation depths, sacrificing the signal to noise ratio during bit detection.

\subsection{Strain Shielding and Confined Plasticity}

Our discussion thus far has been limited to indentations in bulk materials. The formation of piled-up rims has been attributed to the strain, strain capacity, strain rate, and strain hardening susceptibility of the material. In confined systems, a rigid boundary interacting with the stress field during indentation (bit writing) may alter the stress and strain distributions, leading to bulk-deviating mechanical responses [119-122]. For indentations in compliant films, increased rim heights are observed when elastic strain and plastic flow are constrained, or shielded, by a rigid substrate [119-122]. In the case of rigid films on compliant substrates, the plastic yield of the underlying substrate accommodates an enhanced sink-in of the surface around indentation sites [120]. For interfacial systems like TDS, one would expect the material response to scale with any internal rheological gradients (Section 5), in addition to the contribution from the underlying substrate.

The combined influence of rigid dimensional constraints and material anisotropy during indentation has been addressed with high-rate, SPM indentation studies typical of the TDS process [123], and is discussed in the following two sections. A representetive indentation is pictured in Fig. 23.

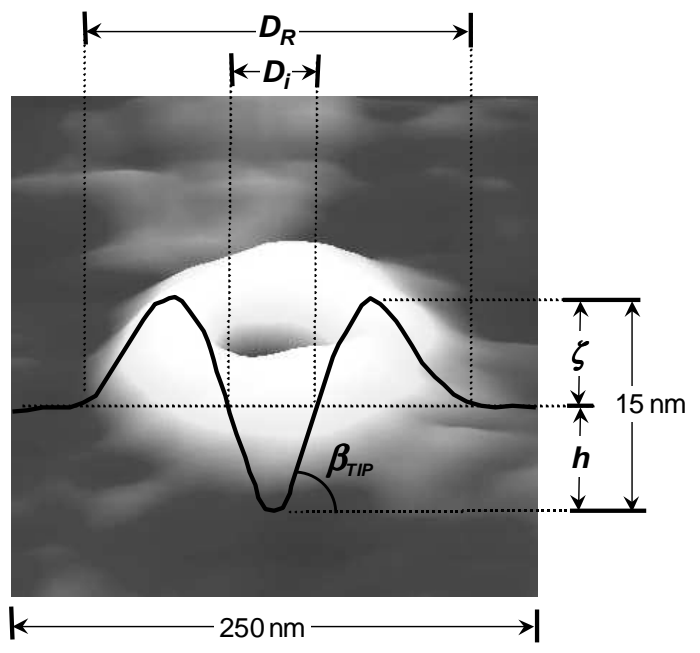

Figure 23. SPM image and geometric evaluation of a residual indentation in polystyrene ( $\operatorname{Rim}$ height, $\zeta$; indentation depth, $h$; $r i m$ diameter, $D_{R} ; \quad$ and indentation diameter, $D_{i}$ ).

\subsubsection{Substrate Constraints}

For indentation mechanics in confined systems, the rim height $\zeta$ is influenced by both process conditions (e.g. normal force, $F_{N}$ ) and geometric conditions (e.g. film thickness). Hence, it is natural that the rim height scales with the indentation depth, i.e. $h\left(F_{N}\right)$, and that substrate constraints are best assessed with respect to ratio of the rim height to the indent depth, $\zeta / h$. In Fig. 24, the load normalized height to depth ratios, $\overline{\zeta / h}$, are reported for indentations in thin polystyrene films $\left(M_{w}=12 k\right)$. For film thicknesses, $\delta$, exceeding $\sim 100 \mathrm{~nm}$, the $\overline{\zeta / h}$ ratio displays a constant value of approximately 0.2 , which reflects the bulk material response. For film thicknesses below $100 \mathrm{~nm}$, the rim height increases with decreasing film thickness. This behavior depends on the substrate material. The rim enhancements were effectively masked by adding a compliant $230 \mathrm{~nm}$ thick crosslinked buffer film (PS-BCB) between the rigid substrate and the indented PS film.

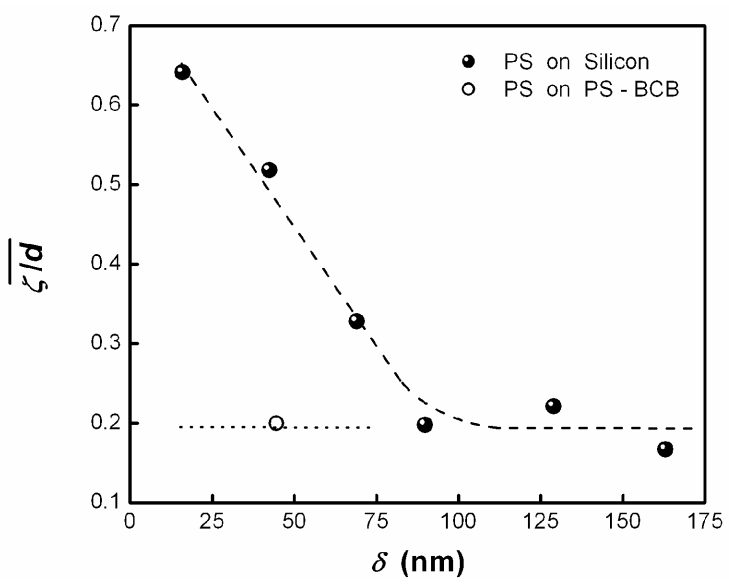

Figure 24: Strain shielding at the substrate interface is evident in the load normalized ratio of rim height to indentation depth $(\overline{\zeta / h})$. For PS on a rigid silicon substrate, rim heights are enhanced for films thinner than $100 \mathrm{~nm}$; a compliant PS-BCB buffer masks the substrate constraints.

Substrate effects during quasi-static indentations, such as enhanced rim heights because of strain shielding, are well known for indentation depths exceeding 10 to $30 \%$ of the film thickness [119-122]. However, the substrate effects in Fig. 24 were observed for residual indent depths significantly less than $10 \%$ of the film thickness. Elastic recovery on unloading may be ruled out [123] based on a quasi-static rheological factor of $X=38$, which exceeds the critical value $(\sim 30)$ for fully plastic deformation [38]. Moreover, for $X=38$, the elastic-plastic simulations of Ramond-Angélélis in Fig. 21, indicate a uniform elastic recovery of roughly $10 \%$ in both the indent depth and diameter [115]. The seemingly far field effects are attributed to the high strain rates $\left(2 \times 10^{3}\right.$ to $\left.1 \times 10^{4} \mathrm{~s}^{-1}\right)$ [123]. These 
rates exceed those of classical quasi-static indentation, and fall within the range of impact dynamics [124]. The main difficulty with impact studies is that the inertial and strainrate effects are usually coupled [125]. The characteristics of wave propagation inevitably depend on the strain-rate dependence of the material properties. At strain rates of $10^{3}-10^{4} \mathrm{~s}^{-1}$, polystyrene succumbs to viscoplastic flow at a nearly constant flow stress of $\sim 20 \mathrm{MPa}$ [126]. This suggests that the propagation speed of a plastic stress wave, $c_{p}(\sigma)=\left(1 / \rho_{\mathrm{o}} \partial \sigma / \partial \varepsilon\right)^{1 / 2}$ (reference [124]), approaches zero above the flow stress. $\rho_{o}$ is the density of the unloaded material, and $\partial \sigma / \partial \varepsilon$ is the slope of the stress-strain curve at a given strain and strain rate. Thus, any plastic stress waves generated in the polymer films are likely to attenuate rapidly [124] (exponentially [127]) as they propagate from the impact site. Consequently, the energy carried by the pressure pulse is dissipated through the plastic deformation processes [127].

Now, let us consider the nature of these plastic deformations, along with any associated interference from the rigid substrate. Based on the indentation cavity model in Fig. 20, it is reasonable assume that the plastically deformed volume is hemispherical with a radius, $C$, equal to one half of the rim diameter, $D_{R}$, in Fig. 23 [38, 122]. Thus, the rim diameter (radius) approximates the penetration depth of plastic deformation. In this case, the appropriate scaling parameter is the ratio of the depth of plastic deformation to the film thickness, $c / \delta$. Under this formalism, the rim heights $(\zeta / h)$ are revisited in Fig. 25 as a function of $c / \delta$.

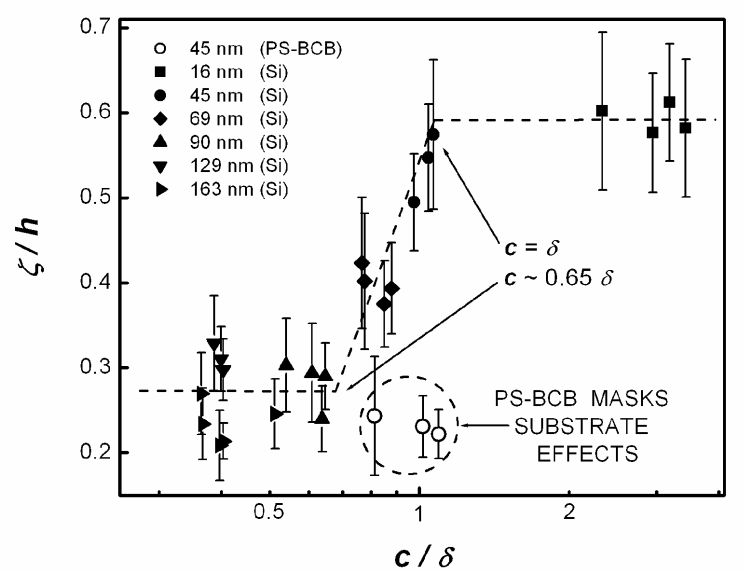

Figure 25: Substrate contribution to strain shielding in thin polystyrene films $(\zeta$ is the rim height, $h$ is the indentation depth, $c$ is the plastic (rim) radius, and $\delta$ is the film thickness; dotted lines are guides).

A sudden increase in the rim height is found when the plastic radius exceeds $\sim 65 \%$ of the film thickness. The rim enhancement results from elastic strain shielding at the rigid substrate, i.e. the material can no longer accommodate strain by elastic expansion in the $-z$ direction. The $\zeta / h$ ratio levels off once the plastic deformation zone comes into direct contact with the rigid substrate. The origin of the plateau at $c / \delta>1$ is not entirely clear, but may arise from geometric changes of the plastic domain boundary (spherical to cylindrical) or from an increasing hydrostatic interaction with the substrate. Again, strain shielding was effectively masked in systems with a PS-BCB buffer film on the silicon substrate, even for plastic zone radii in excess of the film thickness. This suggests that, relative to silicon, the modulus and yield stress of the crosslinked PS-BCB are sufficiently similar to the PS homopolymer to promote a more effective stress distribution across the interface. This can be referred to as a modulus-matched interface. In the absence of modulus-matching, shear stresses will concentrate at the interface [128], potentially activating dewetting instabilities and compromising film stability.

\subsubsection{Structural Anisotropy}

To this point, the competition between material driven length scales and system dimensions emerges with strain shielding at the rigid substrate. Now, let us consider the influence of structural anisotropy in the vicinity of the interface. This discussion is motivated by the findings in Section 5.4, which suggest strain and diffusion induced restructuring over a length scale on the order of $100 \mathrm{~nm}$. The nature of the interfacial $T_{g}$ profiles in Fig. 18 reveals a non-monotonic gradient in the thermomechanical properties. That is, along with the $T_{g}$, the modulus should be expected to follow a similar trend, with a bulk exceeding maximum at approximately $60 \mathrm{~nm}$ from the interface. Beyond this point, the modulus should decrease asymptotically to the bulk value, at roughly 150-200 $\mathrm{nm}$ from the substrate.

An effective modulus may be deduced from the residual indentation geometry by considering the ratio of $p_{m} / \tan \beta$, where the mean contact pressure $p_{m}$ represents the applied stress, and $\tan \beta$ is the residual strain. The pressure $p_{m}$ is defined as $F_{N} / \pi a^{2}$, where $a$ is the contact radius (taken as $1 / 2$ the distance between the rim apices in Fig. 23). The resulting modulus gradient with respect to the film thickness is pictured in Fig. 26 together with the interfacial $T_{g}$ profile for the same material (Fig. 18).

Significant similarities exist between the modulus and $T_{g}$ profiles in Fig. 26. Viewing the glass transition as a mobility barrier, an increase in $T_{g}$ offers resistance to molecular mobility, and the associated rigidity is accompanied by an increase in the modulus. Hence, the individual thermal and mechanical responses should be expected to coincide. The formation of these rheological gradients was discussed in Section 5, and is attributed to shear induced structuring during the spin casting process and to anisotropic diffusion during annealing. The shape of the thermomechanical profile in Fig. 26 implies: for films thicker than $150 \mathrm{~nm}$, the material responds like the bulk; for film thicknesses between $60-120 \mathrm{~nm}$ the surface is more compliant than the immediate sub-surface; and for film thicknesses below $\sim 60 \mathrm{~nm}$, the surface is more rigid than the immediate sub-surface. 


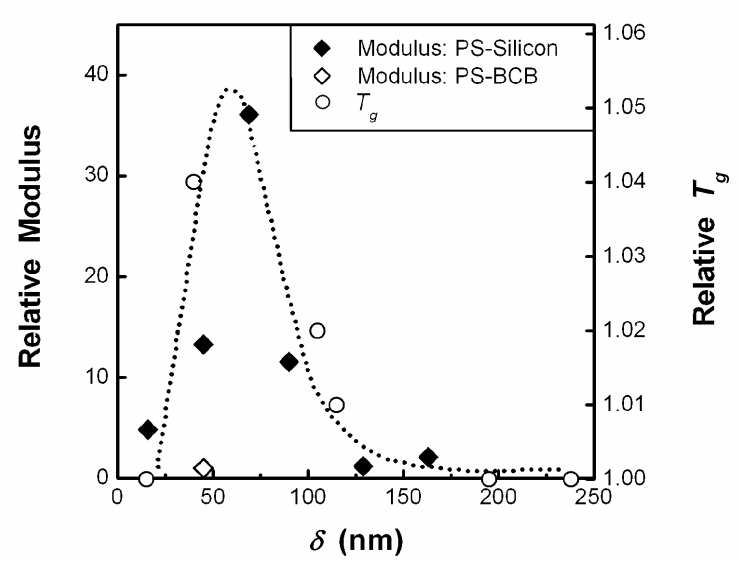

Figure 26. The interfacial thermal and mechanical response profiles for thin polystyrene films are consistent with the rheological boundary layer model in Section 5. The modulus data were determined from indentations with applied loads ranging between 170$190 \mathrm{nN}$, and the $T_{g}$ data are from Fig. 18. The dotted line is drawn as a guide.

Under these conditions, the indentation pressures are distributed between two asymptotic limits: (i) a compliant surface with a rigid sub-surface and (ii) a rigid surface with a compliant sub-surface. For the latter case of a more compliant sub-surface, one would expect a negative rim height, or enhanced sink-in effect.[120] However, it appears that inertial confinement at the rigid substrate counterbalances any sink-in tendencies associated with material anisotropy.

Beyond the immediate implications in thermomechanical storage, our discussion shines a new light on the development of polymer thin film applications. With an understanding of how the interfacial boundary layers are formed (Section 5), and knowledge of how they influence transport operations, the ability to cater interfacial profiles for desired material behaviors offers a new spectrum of design opportunity.

\section{Closing Remarks}

Recent SPM developments provide fundamental insight into mesoscopic dynamical properties that are relevant in nanotechnological applications involving thin films. Two methods have been discussed; shear modulation force microscopy (SM-FM) and friction force microscopy (FFM). The two complementary methods offer the means of tracking thermo-rheological transitions in confined geometries. SM-FM is a convenient and reliable method for determining thermally activated transition points and spatial transition profiles in anisotropic systems. A molecular analysis of the transition process is obtained with FFM. FFM investigations of the dynamics and kinetics in polymer films reveal activation energies related to molecular relaxations, and provide insight to the finite size limitations on structural relaxation near transition points. For instance, direct access to the temperature resolved length scale for cooperative motion during the glass transition is obtained.

Nanotechnological thin film applications, such as the NEMS process for terabit thermomechanical storage, rely on very specific relaxation and transition properties in sub-100 nm systems. Ultimately, achieving the desired performance goals requires materials with a molecular structure that is engineered to function within the system constraints. In polymer films, when film thicknesses are reduced to the sub-100 $\mathrm{nm}$ scale, the structural, material, and transport properties become increasingly dominated by interfacial and dimensional constraints. Rheological boundary layers are formed at interfaces, due to shear induced structuring and anisotropic diffusion during film preparation. Rheological gradients near interfaces lead to bulk-deviating behaviors. These gradients are quantified with interfacial glass transition $\left(T_{g}\right)$ profiles, which provide a molecular structural model of the boundary and allow characterization of the constraints.

Continued thin film optimization and development efforts focus on utilizing interfacial constraints as engineering design opportunities. On the nanoscale, precise material engineering is only possible with an understanding of the polymer dynamics near interfaces. Tailored relaxation properties and enhanced conformational stability may be achieved through control of the interfacial conditions, molecular weight, crosslinking density, and film thickness. Hence, the characterization and control of interfacial boundary layers becomes increasingly important to nanotechnological applications. In NEMS applications that involve thin polymer films, the rheological gradient in the boundary region dictates contact pressures; while the substrate itself can lead to stress and strain shielding at the interface, which compromises film stability. Modulusmatching techniques, i.e. generating a quasi-continuous modulus gradient between opposing faces, offer enhanced interfacial stress transmission and improved stability and durability of the interface. To this end, a resurgence of design methodologies is not impossible, moving from traditional scaling approaches, to a mesoscopic approach where internal rheological gradients are catered to achieve the desired performance characteristics.

\section{Acknowledgements}

We would like to acknowledge Tomoko Gray from the University of Washington, Chemical Engineering Dept. for contributions to Section 4.1. 


\section{Symbols}

a

contact radius, radius of hydrostatic core

$a_{T} \quad$ thermal (horizontal) shift factor

$c$ radius of plastic deformation

$c_{n} \quad$ constant

C contact stiffness calibration factor

$C_{p} \quad$ heat capacity

$D_{i} \quad$ indentation diameter

$D_{R} \quad$ rim diameter

$G \quad$ Gibb's free energy, shear modulus

$G^{\star} \quad$ reduced shear modulus

$E_{*} \quad$ Young's modulus

$E^{*} \quad$ reduced elastic modulus

$E_{A} \quad$ activation energy

$F_{A D H}$ adhesion force

$F_{A P P} \quad$ applied force

$F_{N} \quad$ normal force

$F_{F} \quad$ friction force

$h \quad$ indentation depth,

$H \quad$ enthalpy, tip height

$k_{b} \quad$ Boltzman constant

$k_{T} \quad$ torsional spring constant

$k_{N} \quad$ normal spring constant

$L \quad$ cantilever length

M mass

$n \quad$ reciprocal of work hardening index

$P \quad$ pressure

$p_{m} \quad$ mean contact pressure

$R \quad$ gas constant, contact radius of curvature

$R_{G} \quad$ radius of gyration

$S \quad$ entropy, sensitivity

$T$ temperature

$T_{c} \quad$ critical temperature

$T_{g} \quad$ glass transition temperature

$T_{L} \quad$ cantilever thickness

$T_{M} \quad$ melting temperature

$T_{R} \quad$ reference temperature

$U_{0} \quad$ activation barrier

$V \quad$ volume

$V_{f} \quad$ free volume

$V_{m} \quad$ molar volume

$v \quad$ velocity

$V_{d} \quad$ dewetting velocity

$v_{o} \quad$ characteristic velocity

W cantilever width

$Y \quad$ yield stress

$\alpha \quad$ thermal conductivity

$\beta \quad$ internal damping factor, excluded cone angle

$\delta \quad$ film thickness

$\varepsilon \quad$ strain

$\phi \quad$ stress activation volume

$\gamma \quad$ surface tension

$\Gamma$

$\mu$

$\sigma$

$\tau$

coefficient of friction

stress

shear strength

alpha relaxation time

extrinsic (experimental) time

intrinsic (material) time

contact junction volume

dissipation length

rim height

\section{References}

1. Frommer JE, Overney RM (2001) ACS Symposium Series, vol 781. American Chemical Society, Washington, D.C.

2. Sillescu H (1999) J. Non-Crystalline Solids 243:81

3. Vettiger P, Cross G, Despont M, Drechsler U, Duerig U, Heberle W, Lantz MI, Rothuizen HE, Stutz R, Binnig GK (2002) IEEE Trans. Nanotechnol. 1:39

4. Materials ASfT (ASTM E 1142). American Society for Testing Materials

5. Fried JR (1995) Polymer Science and Technology, edn. Scott, Foresman, and Co., Glenview, IL

6. Eisele U (1990) (Springer-Verlag Berlin Heilelberg, New York) 1990:Chap. 5

7. Arridge RGC (1975) Mechanics of Polymers, edn. Clarendon Press, Oxford

8. Eyring H (1936) J. Chem. Phys. 4:283

9. Williams ML, Landel RF, Ferry JD (1955) J. Am. Chem. Soc. 77:3701

10. Ferry JD (1980) Viscoelastic Properties of Polymers, 3rd edn. John Wiley, New York

11. Bershtein VA, Egorov VM, Kemp TJ (1993) . Ellis Horwood, New York

12. Liu Y, Russell TP, Samant MG, Stohr J, Brown HR, CossyFavre A, Diaz J (1997) Macromolecules 30:7768

13. Frank B, Gast AP, Russel TP, Brown HR, Hawker C (1996) Macromolecules 29:6531

14. Jean JC, Zhang R, Cao H, Yuan J-P, Huang C-M, Nielsen B, Huang C-M (1997) Phys. Rev. B 56:R8459

15. DeMaggio GB, Frieze WE, Gidley DW, Zhu M, Hristov HA, Yee AF (1997) Phys. Rev. Lett. 78:1524

16. Keddie JL, Jones RAL, Cory RA (1994) Europhysics Letters 27:59

17. Forrest JA, Dalnoki-Veress K, Dutcher JR (1998) Physical Review E 58:6109

18. Forrest JA, Svanberg C, Revesz K, Rodahl M, Torell LM, Kasemo B (1998) Physical Review E 58:R1226

19. Richardson H, Sferrazza M, Keddie JL (2003) European Physical Journal E 12:S87

20. Prucker O, Christian S, Bock H, Ruehe J, Frank C, Knoll W (1998) Macromolecular Chemistry and Physics 199:1435

21. Kajiyama T, Tanaka K, Takahara A (1997) Macromolecules 2:280

22. Kajiyama T, Tanaka K, Takahara A (1998) Polymer 39:4665

23. Hammerschmidt JA, Gladfelter WL, Haugstad G (1999) Macromolecules 32:3360

24. Buenviaje C, Dinelli F, Overney RM (2000) In: Frommer J, Overney RM (eds) ACS Symposium Series "Interfacital Properties on the Submicron Scale." Oxford University Press, Oxford

25. Dinelli F, Buenviaje C, Overney RM (2000) Journal of Chemical Physics 113:2043

26. Luginbuhl R, Overney RM, Ratner BD (2001) ACS Symposium: Interfacial Properties on the Submicrometer Scale 781:178

27. Overney RM, Buenviaje C, Luginbuhl R, Dinelli F (2000) J. Therm. Anal. Cal. 59:205

28. Derjaguin BV, Muller VM, Toporov YP (1975) J. Colloid Interface Sci. 53:314

29. Johnson KL, Kendall K, Roberts AD (1971) Proc. Roy. Soc. London A 324:301

30. Greenwood JA (1997) Proc. Roy. Soc. London A 453:1277

31. Greenwood JA, Johnson KL (1998) J. Phys. D: Appl. Phys. 31:3279

32. Maugis D (1992) J. Colloid Interface Sci. 150:243 
33. Barthel E (1998) J. Colloid Interface Sci. 200:7

34. Carpick RW, Ogletree DF, Salmeron M (1999) J. Colloid Interface Sci. 211:395

35. Meyer E, Overney RM, Dransfeld K, Gyalog T (1998) Nanoscience: Friction and Rheology on the Nanometer Scale, edn. World Scientific Publ., Singapore

36. Mindlin RD (1949) J. Appl. Mech. 16:259

37. Pietrement O, Beaudoin JL, Troyon M (1999) Tribology Letters 7:213

38. Johnson KL (1985) Contact Mechanics, Cambridge University Press, Cambridge, UK

39. Buenviaje CK, Ge SR, Rafailovich MH, Overney RM (1998) Mat. Res. Soc. Symp. Proc. 522:187

40. Hutter JL, Bechhoefer J (1993) Rev. Sci. Instrum. 64:1868

41. Cleveland J, Manne S, Bocek D, Hansma PK (1993) Rev. Sci. Instrum. 64:403

42. Sader JE (1995) Rev. Sci. Instrum. 66:4583

43. Neumeister JM, Ducker WA (1994) Rev. Sci. Instrum. 65:2527

44. de Gennes PG (2000) C.R. Acad. Sci. IV:1

45. Ge S, Pu Y, Zhang W, Rafailovich M, Sokolov J, Buenviaje C, Buckmaster R, Overney RM (2000) Phys. Rev. Lett. $85: 2340$

46. Buenviaje C, Dinelli F, Overney RM (2000) In: Frommer JE, Overney RM (eds) Interfacial Properties on the Submicron Scale, vol 781. American Chemical Society, Washington, DC p 76

47. Schallamach A (1971) Wear 17:301

48. Overney RM, Takano H, Fujihira M, Paulus W, Ringsdorf H (1994) Phys. Rev. Lett. 72:3546

49. Briscoe BJ, Evans DCB (1982) Proc. R. Lond. A 380:389

50. He M, Szuchmacher-Blum A, Overney G, Overney RM (2002) Phys. Rev. Lett. 88:154302

51. Gnecco E, Bennewitz R, Gyalog T, Loppacher C, Bammerlin M, Meyer E, Güntherodt H-J (2000) Phys. Rev. Lett. 84:1172

52. Dudko OK, Filippov AE, Klafter J, Urbakh M (2002) Chemical Physics Letters 352:499

53. Sang Y, Dube M, Grant M (2001) Phys. Rev. Lett. 87:174301/1

54. Heslot F, Baumberger T, Perrin B, Caroli B, Caroli C (1994) Phys. Rev. E 49:4973

55. Rozman MG, Urbakh M, Klafter J (1998) Physica A 249:184

56. Sills S, Overney RM (2003) Phys. Rev. Lett. 91:095501

57. Metzler R, Klafter J (2000) Phys. Reports 339:1

58. Metzler R, Klafter J (2001) Physica A 302:290

59. Sokolov IM (2000) Phys. Rev. E 63:011104/1

60. Luedtke WD, Landman U (1999) Phys. Rev. Lett. 82:3835

61. He M (2002) Material and transport properties of fluids in nanoconfining systems, $\mathrm{PhD}$, University of Washington

62. Pokrovskii VN (2000) Mesoscopic theory of polymer dynamics, Kluwer Academic Publishers, Dordrecht

63. Luo J, Haller M, Ma H, Liu S, Kim TD, Tian Y, Chen B, Jang S-H, Dalton LR, Jen AK-Y (2004) J. Phys. Chem. B 108:8524

64. Kulkarni AP, Jenekhe SA (2003) Macromolecules 36:5285

65. Grosch KA (1963) Proc. R. Soc. London Ser. A 274:21

66. Ludema KC, Tabor D (1966) Wear 9:329

67. Schallamach A (1953) Wear 6:375

68. Kontorova T, Frenkel YI (1938) Zh. Eksp. Teor. Fiz. :8

69. Chernyak YB, Leonov AI (1986) Wear 108:105

70. Ronsin O, Coeyrehourcq KL (2001) Proceedings of the Royal Society of London Series a-Mathematical Physical and Engineering Sciences 457:1277
71. Vorvolakos K, Chaudhury MK (2003) Langmuir 19:6778

72. Reich S, Eisenberg A (1972) J. Poly. Sci. A-2 10:1397

73. Yano O, Wada Y (1971) J. Poly. Sci. A-2 9:669

74. Boyer RF, Turley SG (1978) In: D.J.Meier (ed) Molecular basis of transitions and relaxations . Gordon and Breach Science Publishers, New York p 333

75. Debenedetti PG, Stillinger FH (2001) Nature 410:259

76. Biju VP, Ye JY, Ishikawa M (2003) Journal of Physical Chemistry B 107:10729

77. Adam G (1965) J. Chem. Phys. 43:139

78. Ediger MD (2000) Annu. Rev. Phys. Chem. 51:99

79. Richert R (2002) J. Phys.: Condens. Matter 14:R703

80. Cohen MH, Grest GS (1979) Phys. Rev. B 20:1077-1098

81. Tracht U, Wilhelm M, Heuer A, Feng H, Schmidt-Rohr K, Spiess HW (1998) Phys. Rev. Lett. 81:2727

82. Sillescu H, Bohmer R, Diezemann G, Hinze G (2002) Journal of Non-Crystalline Solids 307:16

83. Berthier L (2004) Phys. Rev. Lett. 69:020201(1

84. Roland CM, Casalini R (2003) J. Chem. Phys. 119:1838

85. Hedvig P (1977) Dielectric Spectroscopy of Polymers, edn. John Wiley and Sons, New York

86. Bennemann C, Donati C, Baschnagel J, Glotzer S (1999) Nature 399:246

87. Lyulin AV, de Groot JJ, Michels MAJ (2003) Macromol. Symp. 191:167

88. Sills S, Overney RM (2005) Phys. Rev. Lett. (submitted)

89. Sills SE, Overney RM, Chau W, Lee VY, Miller RD, Frommer J (2004) J. Chem. Phys. 120:5334

90. Overney RM, Guo L, Totsuka H, Rafailovich M, Sokolov J, Schwarz SA (1997) In: Drake JM, Klafter J, Kopelman R (eds) Dynamics in Small Confining Systems IV , vol 464. Mat. Res. Soc., Pittsburgh, PA p 133

91. Rabeony M, Pfeiffer DG, Behal SK, Disko M, Dozier WD, Thiyagarajan P, Lin MY (1995) J. Che. Soc. Faraday Trans. 91:2855

92. Zheng X, Rafailovich MH, Sokolov J, Strzhemechny Y, Schwarz SA, Sauer BB, Rubinstein M (1997) Phys. Rev. Lett. 79:241

93. Overney RM, Leta DP, Fetters LJ, Liu Y, Rafailovich MH, Sokolov J (1996) J. Vac. Sci. Technol. B 14:1276

94. Buenviaje C, Ge S, Rafailovich M, Sokolov J, Drake JM, Overney RM (1999) Langmuir 15:6446

95. Chen HP, Katsis D, Mastrangelo JC, Chem SH, Jacobs SD, Hood PJ (2000) Advanced Materials 12:1283

96. Dalnoki-Veress K, Forrest JA, Gennes PGd, Dutcher JR (2000) J. Phys. IV 10:221

97. Forrest JA, Mattsson J (2000) Physical Review E 61:R53

98. Tseng KC, Turro NJ, Durning CJ (2000) Physical Review E 61:1800

99. Kim JH, Jang J, Zin WC (2001) Langmuir 17:2703

100. Brogly M, Bistac S, J. S (1998) Macromol. Theor. Simul. $7: 65$

101. Orts WJ, Van Zanten JH, Wu WL, Satija SK (1993) Phys. Rev. Lett. 71:867

102. Saito S, Hashimoto T (2001) J. Chem. Phys. 114:10531

103. Morfin I, Linder P, Boue F (1999) Macromolecules 32:7208

104. Wu X, Squires KD (2000) J. Fluid Mech. 418:231

105. Wang CL, Wang SJ (1995) Phys. Rev. B 51:8810

106. Binnig GK, Cherubini G, Despont M, Duerig UT, Eleftheriou E, Vettiger P (2004) In: Bhushan B (ed) Springer Handbook of Nanotechnology . Springer-Verlag, Heidelberg, Germany

107. Karapanagiotis I, Evans DF, Gerbrich WW (2002) Polymer 43:1343 
108. Bushan B (1999) Principles and Applications of Tribology, edn. John Wiley \& Sons, New York

109. Hill (1950) The mathematical theory of plasticity, edn. Oxford University Press, London

110. Johnson KL (1970) J. Mech. Phys. Solids 18:115

111. Tabor D (1951) The hardness of Metals, edn. Oxford University Press, London

112. Briscoe BJ, Evans PD, Biswas SK, Sinha Sk (1996) Trib. Int. 29:93

113. Adams MJ, Allan A, Briscoe BJ, Doyle PJ, Gorman DM, Johnson SA (2001) Wear 251:1579

114. Jardret VD, Oliver WC (2000) Mat. Res. Soc. Symp. Proc. 594:251

115. Ramond-Angélélis C (1998) Analyse mécanique des essais d'indetation sur matériaux élasto-plastiques homogènes ou multi-couches. Application à la caractérisation de la rhéologie et de la tenue mécanique des films minces, Ecole Nationale Supérieure des Mines de Paris

116. Vaisyanathan R, Dao M, Ranichandran G, Suresh S (2001) Acta Mater. 49:3781
117. Malow TR, Koch CC, Miraglia PQ, Murty KL (1998) Mat. Sci. Eng. A252:36

118. Matthews JR (1980) Acta Met. 28:311

119. Tsui TY, Pharr GM (1999) J. Mater. Res. 14:292

120. Tsui TY, Vlassak J, Nix WD (1999) J. Mater. Res. 14:2204

121. Randall NX, Julia-Schmutz C, Soro JM (1998) Surface and Coatings Tech. 108-109:489

122. Kramer DE, Volinsky AA, Moody NR, Gerberich WW (2001) J. Mater. Res. 16:3150

123. Sills SE, Overney RM, Gotsmann B, Frommer J (2005) Tribo. Lett. (in press)

124. Zukas JA, Nicholas T, Swift HF, Greszczuk LB, Curran DR (1982) Impact Dynamics, edn. John Wiley \& Sons, New York

125. Wang LL (2003) Chinese J. of Mech. A 19:177

126. Walley SM, Field JE, Pope PH, Safford NA (1989) Philo. Thans. Roy. Soc. London. Series A 328:1

127. Cristescu N (1967) Dynamic Plasticity, edn. North-Holland Publishing Co., Amsterdam

128. Giannakopoulos AE (1998) Thin Solid Films 332:172 